Supporting Information for:

\title{
Charge Transfer Excitation and Asymmetric Energy Transfer at the Interface of Pentacene-Perfluoropentacene Heterostacks
}

\author{
Anna-Katharina Hansmann ${ }^{\dagger}$, Robin C. Döring ${ }^{\ddagger}$, Andre Rinn ${ }^{\ddagger}$, Steffen M. Giesen ${ }^{\dagger}$, Melanie Fey ${ }^{\ddagger}$, \\ Tobias Breuer ${ }^{\S}$, Robert Berger ${ }^{* \dagger}$, Gregor Witte ${ }^{* \S}$, and Sangam Chatterjee ${ }^{* \ddagger}$
}

\footnotetext{
${ }^{\dagger}$ Department of Chemistry, Philipps-University Marburg, Hans-Meerwein-Straße 4, D-35032 Marburg, Germany

‡Institute of Experimental Physics I and Center for Materials Research (LaMa), Justus-Liebig-University Giessen, Heinrich-Buff-Ring 16, D-35392 Giessen, Germany

$\S$ Department of Physics and Materials Sciences Center, Philipps-University Marburg, Renthof 7, D-35032 Marburg, Germany
}

*E-mails: sangam.chatterjee@physik.uni-giessen.de gregor.witte@physik.uni-marburg.de robert.berger@chemie.uni-marburg.de

\section{Contents}

S1. Structural Characterization ......................................................... 2

S2. Absorption Spectra of the Unitary Films ........................................... 3

S3. Orbital Phase Convention ..................................................... 4

S4. Additional Theoretical Results .................................................. 6 


\section{S1. Structural Characterization}

All samples were analyzed by X-ray diffraction (XRD) and, in the case of the lying heterostack, also using Near-Edge-X-Ray-Absorption-Fine-Structure-Spectroscopy (NEXAFS). A full analysis of the data for the heterostack is presented in Ref. [40], hence we will discuss only the most relevant outcomes of the structural analysis here. A specular XRD scan shows that the PEN molecules in the bottom layer exhibits crystalline arrangements in the (1-1-2) and (022) orientations of the PEN Siegrist-phase, both of which correspond to lying molecular orientations (yet, with a slight molecular rotation along the long axis which is a result of the herringbone arrangement in the PEN crystal structure). As the XRD scans furthermore reveal, the PFP molecules in such films (where PFP is deposited on top of PEN) crystallize in the $\pi$ stacked polymorph (PSP) of PFP, in the (002) orientation which corresponds to an arrangement where all molecules are aligned with their backbones parallel to the substrate. Consequently, we can safely conclude that the molecules of both species are aligned in a parallel fashion. To furthermore verify that the molecules are aligned parallel also at the interface, we have performed NEXAFS measurements of very thin PFP films (about $2 \mathrm{~nm}$ ) on top of PEN layers. The angle-resolved NEXAFS measurements show a very clear dichroism for the spectral contributions of PEN as well as PFP, exhibiting largest intensity under grazing incidence and strongly reduced intensity under normal incidence which again corresponds to a lying molecular orientation of both species. The quantitative analysis yielded effective tilt angles of the molecular $\pi$-system of $30^{\circ}$ for Pen and less than $26^{\circ}$ for PFP. We note that NEXAFS spectroscopy is a highly surface sensitive method, which therefore exclusively probes the orientation in the uppermost layers, i.e. at the interface and is not influenced by contributions from deeper layers (details of the analysis are given in Ref. [40]).
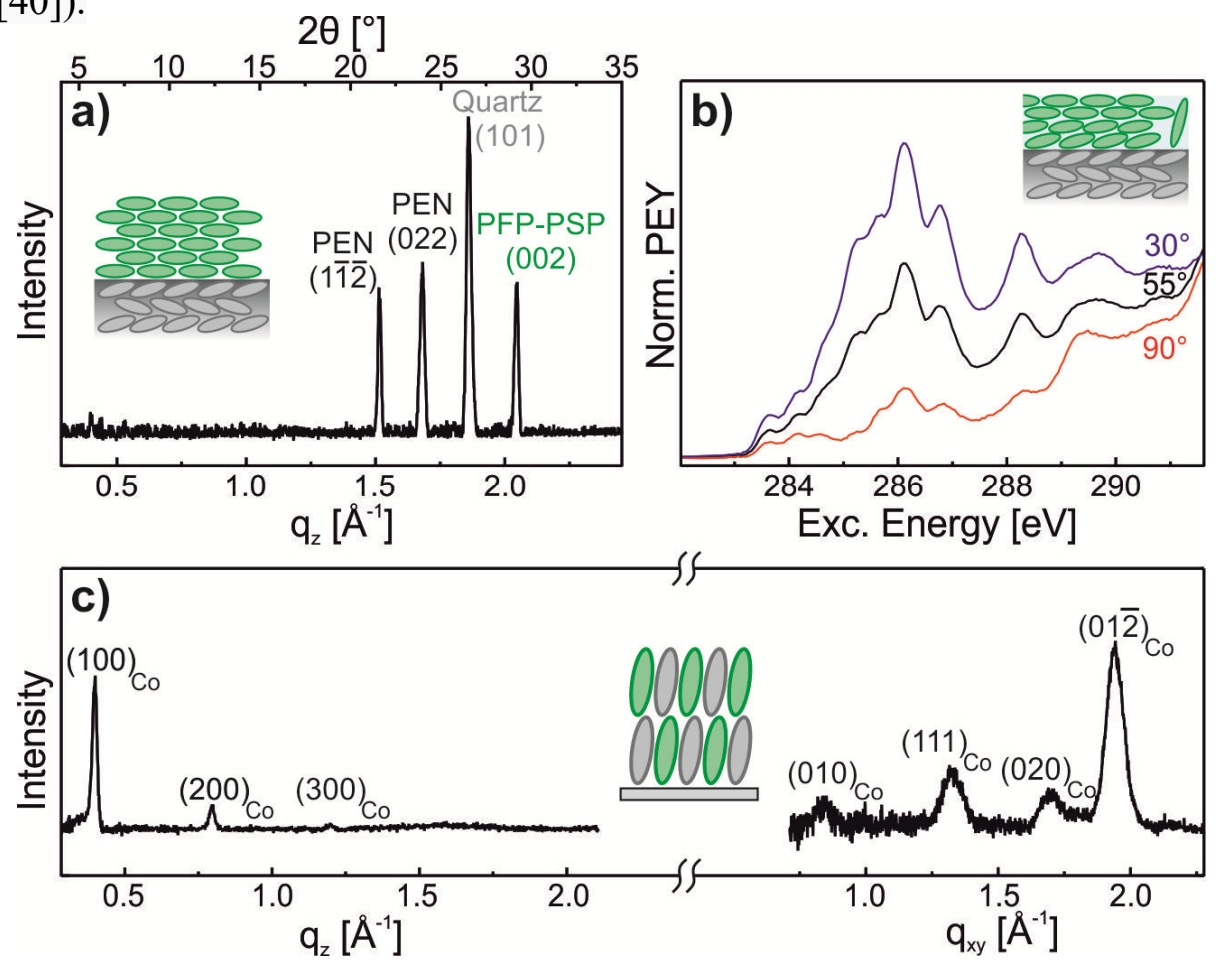

Figure S1. (a) Specular X-ray diffractogram of a PFP/PEN stack with nominal thickness of 20nm in the bottom PEN layer and the PFP top layer grown on graphene sheet supported by a quartz substrate. (b) $\mathrm{C} 1 \mathrm{~s}$ NEXAFS spectra of the initial growth (2nm) of PFP grown on a $20 \mathrm{~m}$ PEN film on graphene. c) XRD data of a coevaporated PEN:PFP film with equimolar mixing ratio and a total film thickness of $40 \mathrm{~nm}$, specular X-ray diffractogram (left) and in-plane GIXD measurements (right). All XRD data are measures with $\mathrm{Cu}$ K $\alpha$ radiation. 
The specular XRD scans of the sample prepared by simultaneous equimolar deposition of PEN and PFP show signals at $\mathrm{q}_{\mathrm{z}}$-values of $0.40 \AA^{-1}, 0.80 \AA^{-1}$ and $1.21 \AA^{-1}$. Since the vertical lattice spacing for PEN, PFP and PEN:PFP crystalline layers (with upright molecular orientation) is nearly identical, the specular measurements do not directly allow to conclude that the codeposited film is actually intermixed on a molecular level. Therefore, we have also performed GIXD measurements. The corresponding data shows signals at $\mathrm{q}_{\mathrm{xy}}$ values of $0.87 \AA^{-1}, 1.39 \AA^{-1}, 1.74 \AA^{-1}$ and $1.93 \AA^{-1}$. These values are in very good agreement with previously reported data for PEN:PFP samples [S1, S2] and clearly different from signals expected for the individual compounds, hence proving that the sample is indeed intermixed on a molecular level. Comparing the observed data with the previously reported crystal structure [26], we have assigned the observed signals to the (010), (111), (020) and (01-2) lattice planes in the PEN:PFP heterostructure Combining these information, we can safely conclude that this sample is formed by molecules in upright molecular orientation, which are intermixed on a molecular scale.

[S1] Salzmann et al. Langmuir 24, 14 (2008).

[S2] Hinderhofer et al. J. Chem. Phys. 134, 104702 (2011).

\section{S2. Absorption Spectra of the Unitary Films}

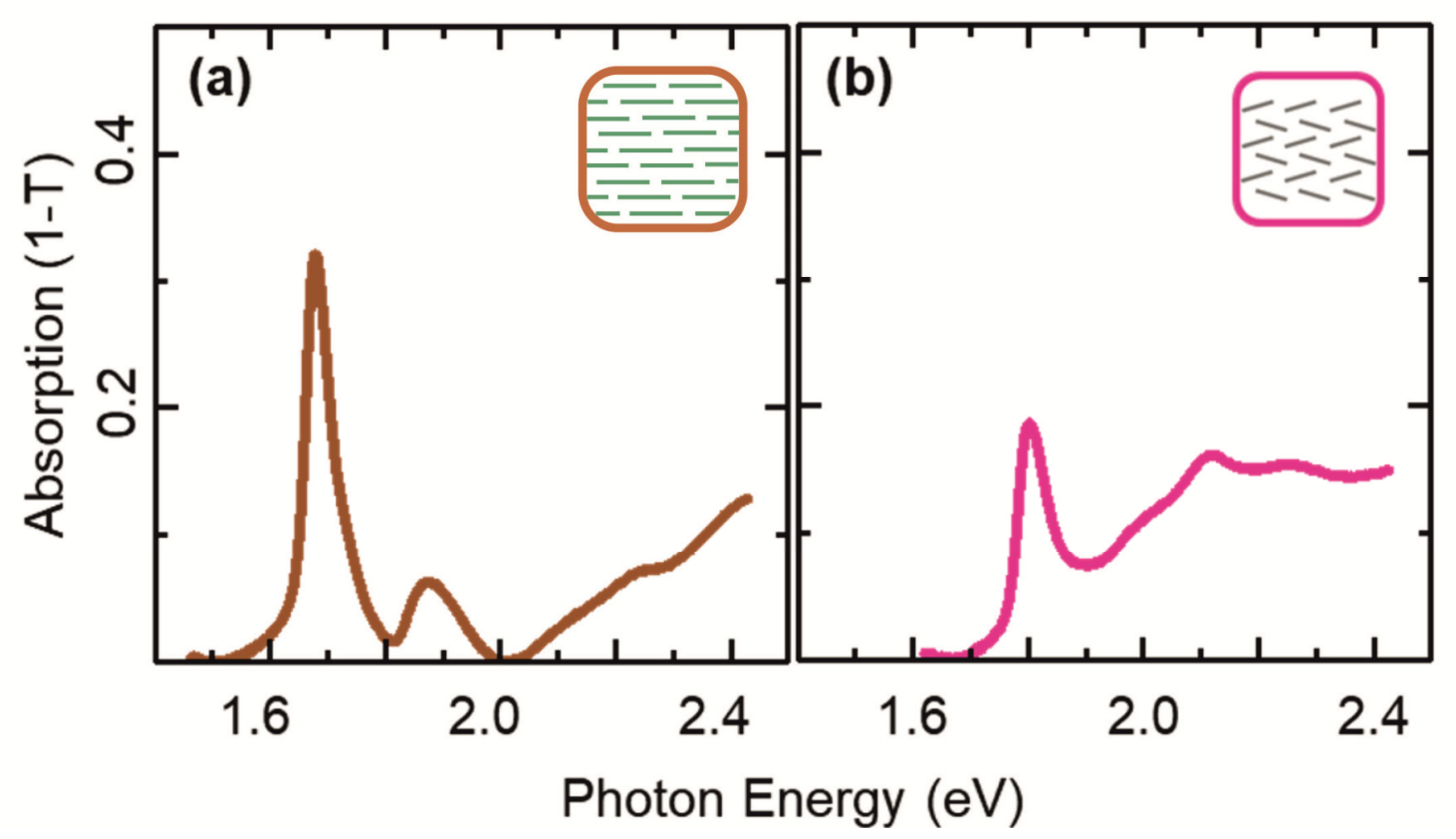

Figure S2. Absorption spectra for unitary PFP (a) and PEN (b) thin films of $20 \mathrm{~nm}$ film thickness and lying molecular orientation. The measurements have been performed at $20 \mathrm{~K}$ lattice temperature. 


\section{S3. Orbital Phase Convention}

To allow an interpretation of the sign of the coupling matrix elements, the phase of the individual orbitals has to be maintained consistently. Here, we aligned the phases of all orbitals of the excited and ionic determinants to those of the corresponding ground state determinant using the orbital overlap to identify correspondence and phase changes. The contribution to the matrix elements was determined to depend almost exclusively on the HOMOs and LUMOs. Their phases were manually kept consistent between the different dimer structures. Because of the similarity in the structure and orbitals of PEN and PFP, also the orbitals for both of the monomers in each dimer could be kept consistent. This can be imagined as only allowing a translation of the orbitals from one monomer to the other, but not a rotation exceeding $90^{\circ}$.

The orbitals are shown in Fig. S3a for qualitative comparison of the phase on the example of the PEN HOMO and LUMO, but for convenience, we report for the structures in Fig. S3b,c,d also the phases used for HOMO and LUMO of the building blocks.
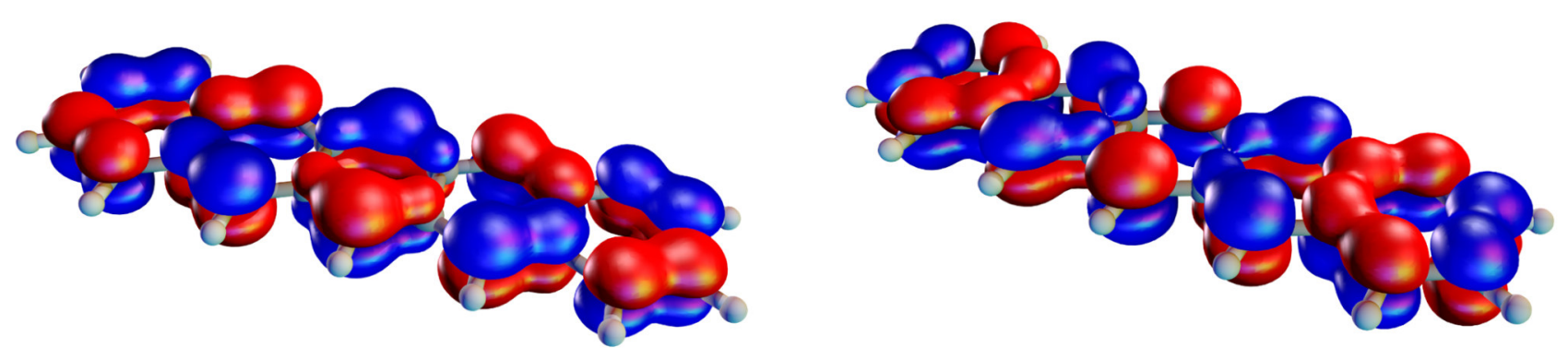

Figure S3a. The HOMO (left) and LUMO (right) of PEN as a qualitative guide to the applied orbital phase convention
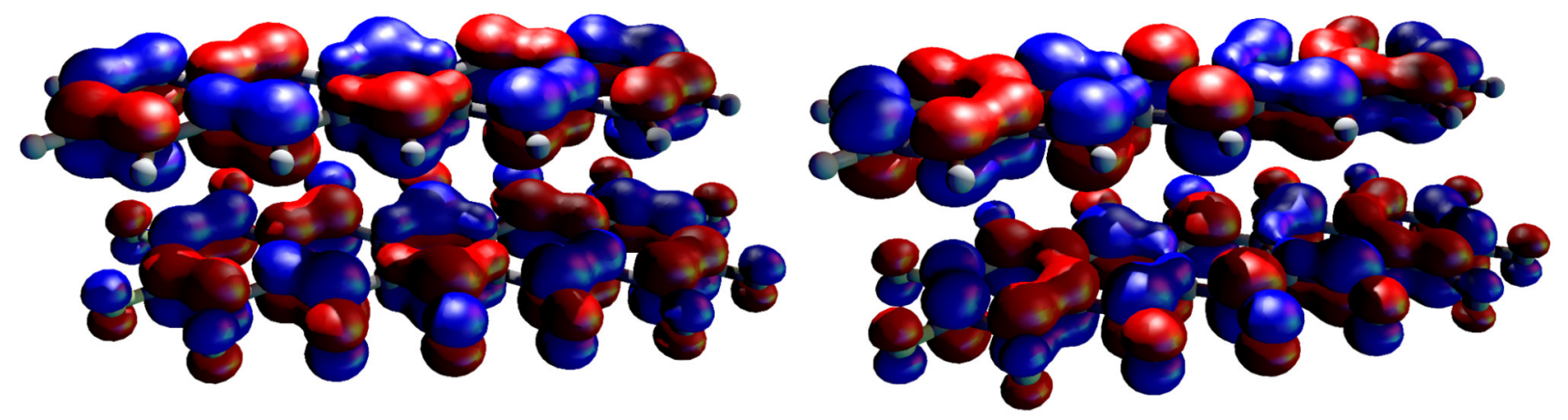

Figure S3b. The HOMOs (left) and LUMOs (right) of the building blocks in the $\pi$-stacked dimer arrangement 

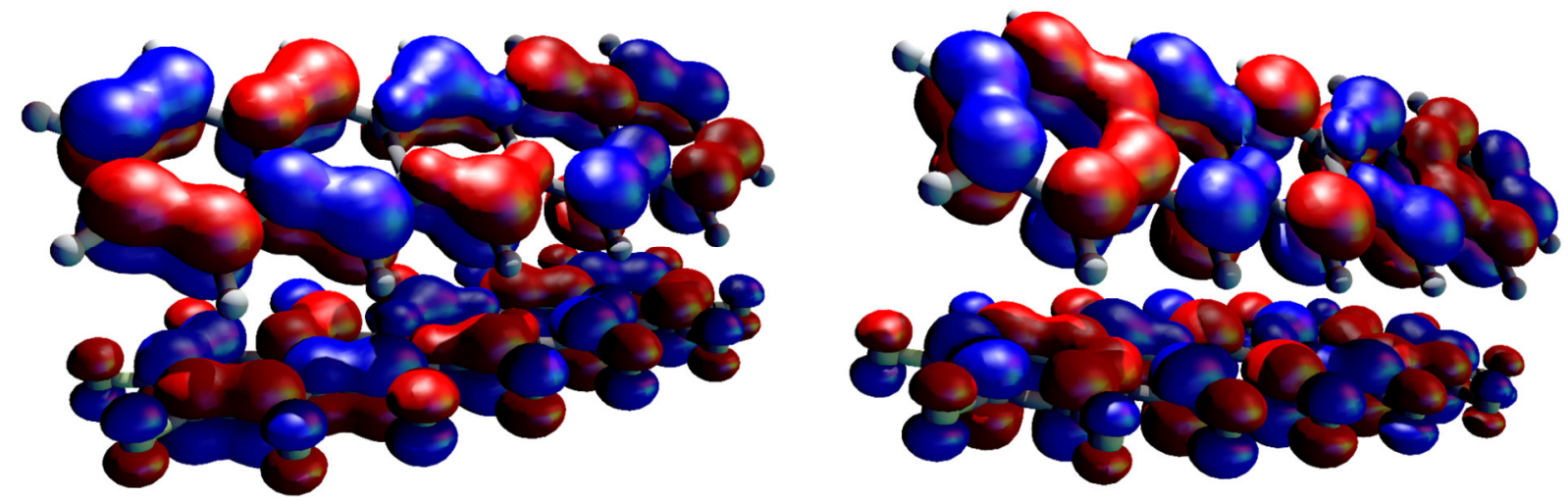

Figure S3c. The HOMOs (left) and LUMOs (right) of the building blocks in the herringbone dimer arrangement.

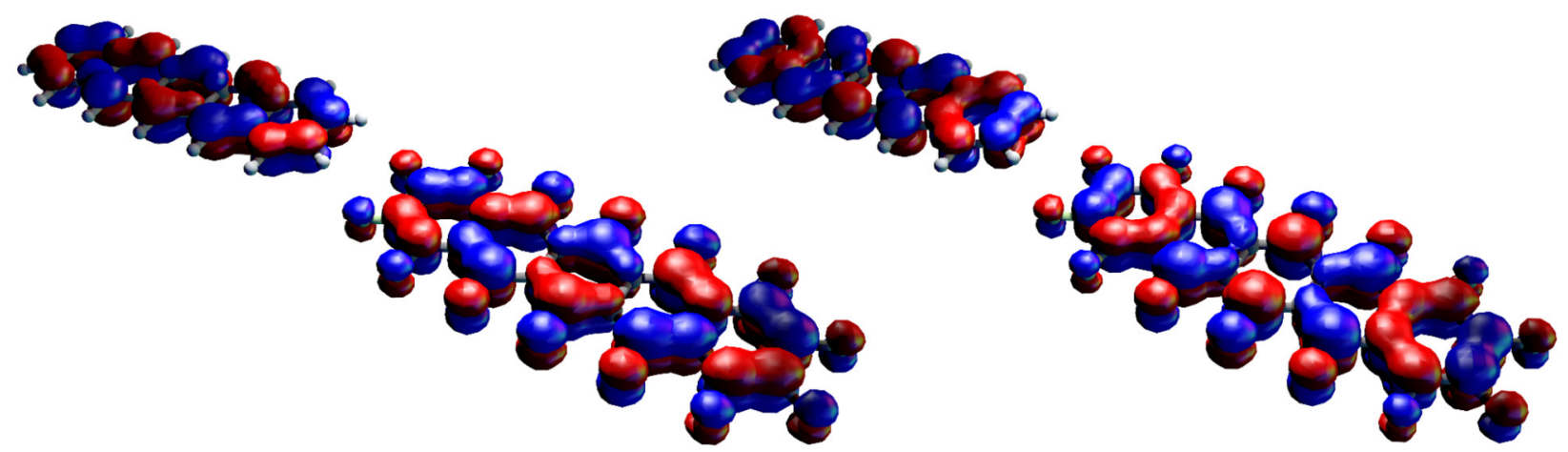

Figure S3d. The HOMOs (left) and LUMOs (right) of the building blocks in the end-on dimer arrangement. 


\section{S4. Additional Theoretical Results}

Bulk and interface regions were simulated by extending the dimer antisymmetrized product wave functions of Eq. (2) to a one dimensional hexamer model $\left|\Phi_{\mathrm{Pen}_{1}}^{m_{1}} \Phi_{\mathrm{Pen}_{2}}^{m_{2}} \Phi_{\mathrm{Pen}_{3}}^{m_{3}} \Phi_{\mathrm{PFP}_{4}}^{m_{4}} \Phi_{\mathrm{PFP}_{5}}^{m_{5}} \Phi_{\mathrm{PFP}_{6}}^{m_{6}}\right\rangle$ as prescribed by Eq. (3) (see Computational Methods section of the main text). Here, the monomer at index $i$ is in state $m_{i}$ with $1 \leq i \leq 6$. To model the movement of the exciton from the bulk to the interface, the states were prepared so that the excitation was localized to the $k$-th monomer. Then, $m_{k}$ is the first excited singlet state and $m_{i}$ the ground state for all $i \neq k$. The results here also indicate an activation barrier for PFP, which is of similar magnitude as for HOMO-LUMO energy differences schematically shown in Figure $4 \mathrm{~b}$ of the main text. Moreover, in this model there even is a slight decrease in energy for the excitation localized at the PEN monomer positioned directly at the interface, indicating a transfer of the excitation from the PEN bulk to the interface without any activation barrier. Note that further improvements of the theoretical model are in principle possible, for instance by accounting for polarization effects, by improving the treatment of orbital relaxation within a self-consistent group function SCF approach or by inclusion of additional electron correlation effects in the description of the building blocks.

To get a better picture of boundary, bulk and interface regions, we also calculated the diabatic excitation energies for a dodecamer (12 monomers) of another theoretical model system: An ethylenetetrafluorethylene (ETH-TFE) slipped $\pi$-stack was used, the smallest corresponding $\pi$-system and perfluorinated derivative. The result is similar to that obtained for the Pen-PFP interface, but due to a switch in the orbital energies, the energy gradient changes direction as well. This does not inhibit the visibility of the boundary and interface influences on the extended system. This information is then directly transferable to the PEN-PFP system, with PEN showing the same overall behavior as TFE, and PFP the same as ETH. The plot of the excitation energies and orbital energy differences for the PEN-PFP hexamer is shown in Figure S4 on the left, the excitation energies for the ETH-TFE model system on the right.

For both systems, the calculation were repeated at $80 \%, 90 \%$ and $110 \%$ of the intermolecular COM distance. For PEN-PFP, 90\% corresponds to the optimzed dimer distance using the CAM-B3LYP functional, $80 \%$ is slightly below the distance found in the co-crystal structure for the corresponding dimer subsection. The calculations show that a smaller distance leads to a higher barrier to move from the bulk to the interface region for PFP and ETH. The amount of change is shown more clearly in Fig. S5 for both systems. For ETH-TFE a, exponential increase is observed, while for PEN-PFP, the influence is more linear, which can be attributed to geometric reasons and the opposing effects of the interface and boundary region in the smaller hexamer model. The extent of the interface and boundary regions on the excitations 
energies is visible in the ETH-TFE dodecamer model. Removing the influence of the boundary region on the bulk region by employing a larger model system would thus likely show a stronger increase in barrier for smaller intermolecular distances.
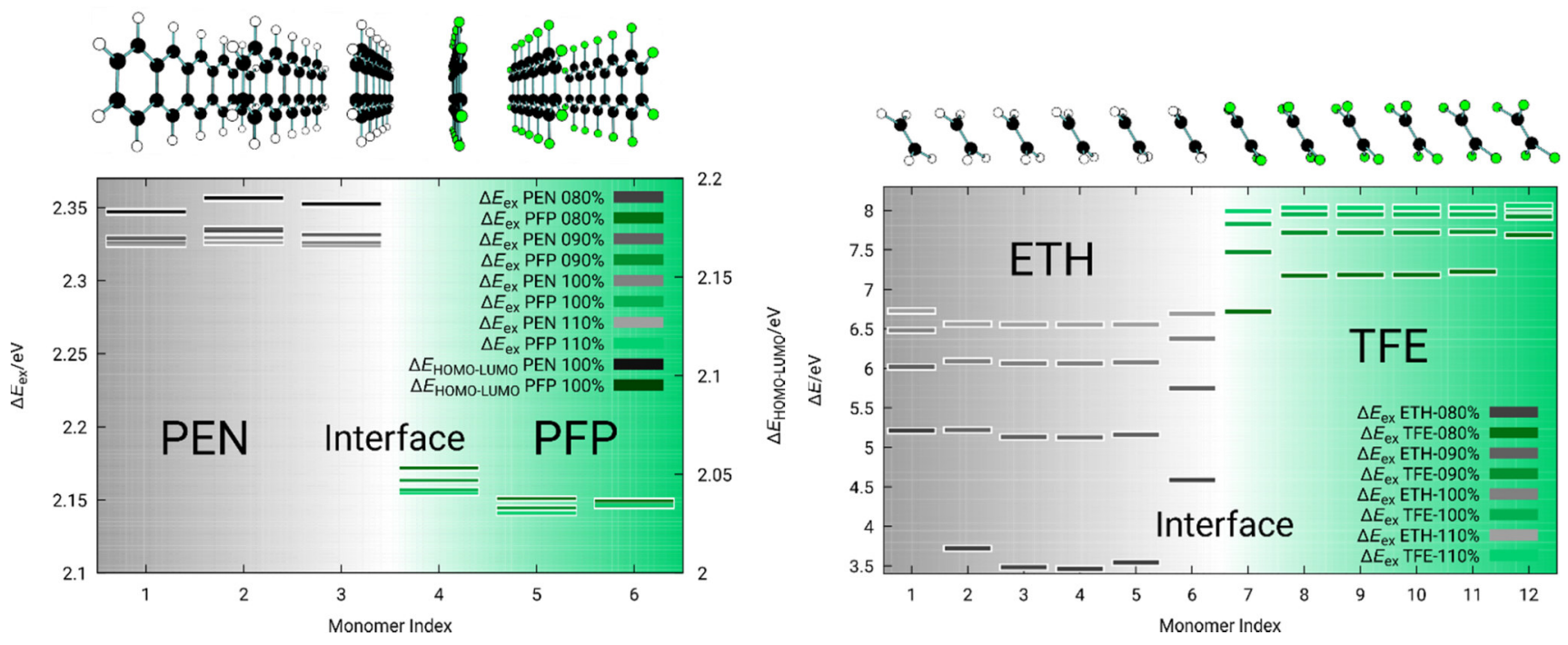

Figure S4. On the left, the diabatic excitation energies and HOMO-LUMO energy differences in the hexamer model of the PEN-PFP interface are displayed for different intermolecular distances of the monomers. The excitation energies are shown on the left axis, the orbital energy differences on the right axis, both in eV. The orbitals assigned to monomers 1 and 2 as well as 5 and 6 are partially delocalized, but can still be mostly attributed to one distinct molecule. On the right, the diabatic excitation energies in a dodecamer model of the ethylene-tetrafluorethylene (ETH-TFE) interface are depicted for different intermolcular distances. The excitation energies are shown in eV. For both subfigures the intermolecular distances are given in percent of the B3LYP optimized COM distance. A reduction of the distance to $90 \%$ corresponds to the distance obtained from the CAM-B3LYP optimized dimer geometry. At $80 \%$, the distance is slightly below that of the corresponding dimers of the co-crystal structure.
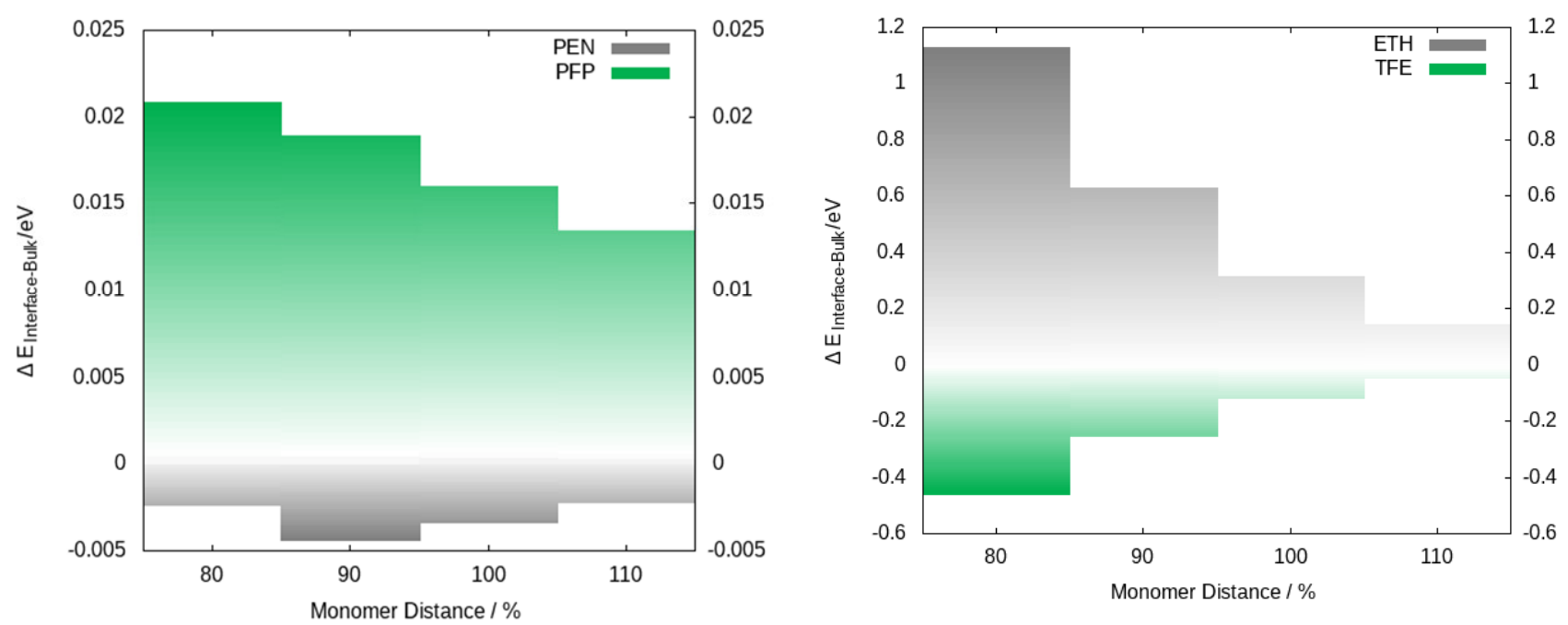

Figure S5. Change in energetic barrier between bulk and interface region for PEN-PFP (left) and ETHTFE (right) depending on the intermolecular COM distance. Here, 100\% corresponds to the B3LYP optimized structure (dimer distance). 


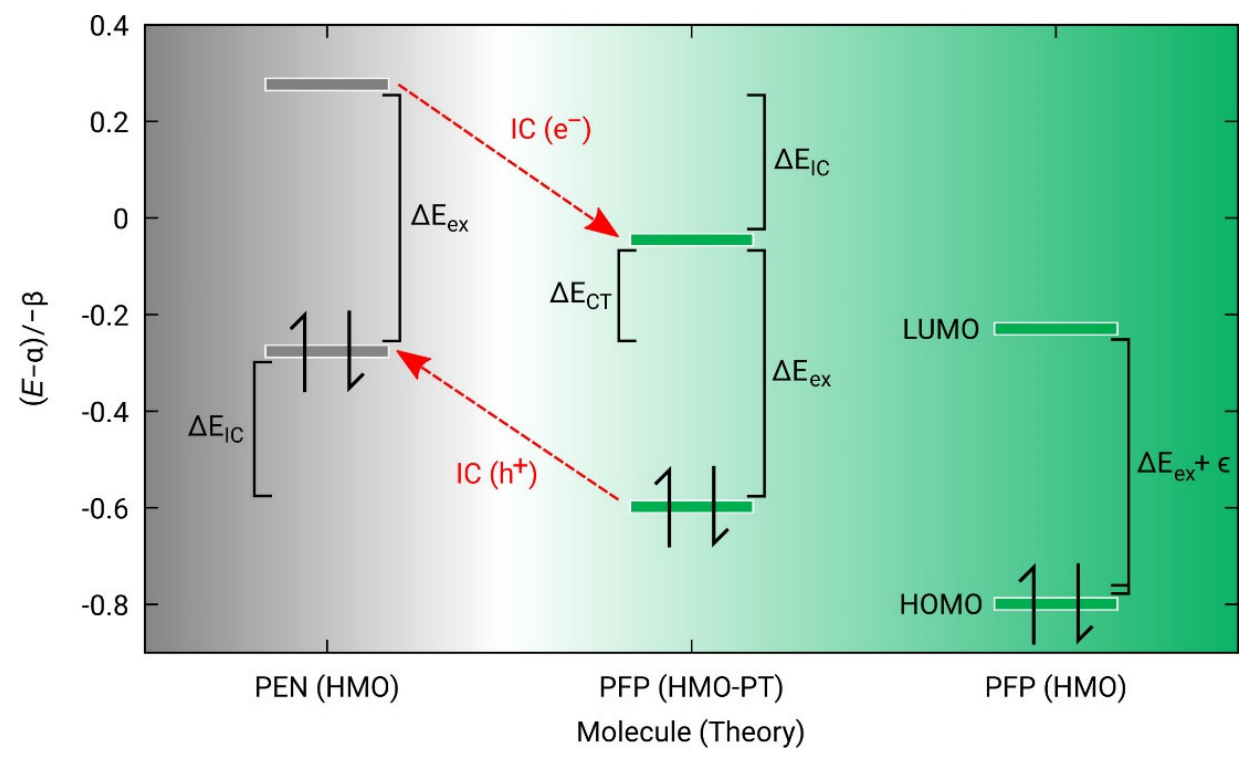

Figure S6. HOMO and LUMO energies obtained within HMO theory. To stress the qualitativeness of these results, the energies are given in units of the resonance integral $\beta$ and reduced by the Coulomb integral $\alpha$. The PEN orbitals are unperturbed HMO calculations for the system. The PFP (HMO-PT) orbitals are obtained using first order HMO perturbation theory, and thus show the same HOMO-LUMO gap as for PEN. Only for the exact HMO calculation of PFP, an, albeit small, change in the gap is introduced. Conversely, the total shift of the orbital energies leads to a significantly different intermolecular energy gap between an exemplary PEN donor HOMO and a PFP acceptor LUMO. This is the CT energy, which is significantly lower than the local singlet excitation.

The Cartesian coordinates (in Ångström) for the dimer structures, energy-minimized with the program package Turbomole as described in the main text, are given in Table S1 to S3. The hexamer structure is given in Table S4. Note that the numerical results are given for completeness and to ensure reproducibility beyond physical interpretation.

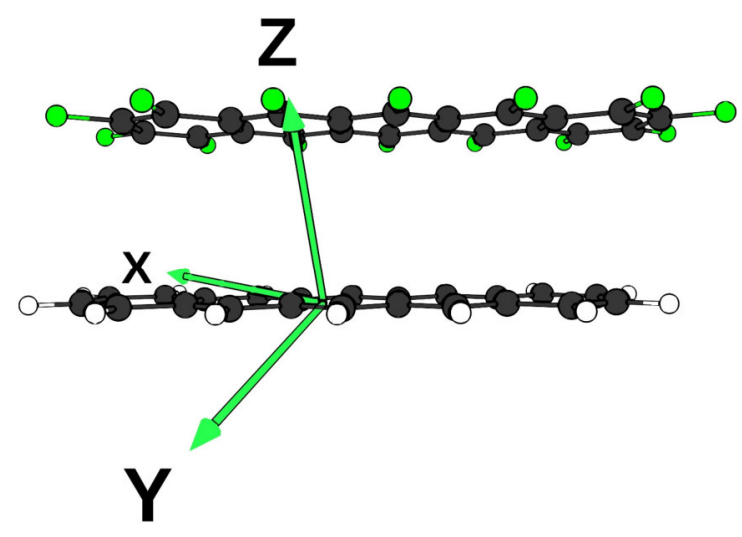

Figure S7. Plot of the Cartesian coordinates given in Table S1 for the PEN-PFP $\pi$-stacked dimer. 
Table S1. Cartesian coordinates of a $\pi$-stacked PEN-PFP dimer in Ångstrom.

\section{Element symbol}

\section{$\mathbf{X}$}

\section{$\mathbf{Y}$}

\begin{tabular}{|c|}
\hline $\mathbf{C}$ \\
\hline $\mathbf{C}$ \\
\hline $\mathbf{C}$ \\
\hline $\mathbf{C}$ \\
\hline $\mathbf{C}$ \\
\hline
\end{tabular}

\begin{tabular}{|r}
\hline $\mathrm{C}$ \\
\hline $\mathrm{C}$ \\
\hline $\mathrm{C}$
\end{tabular}

\begin{tabular}{|r}
\hline C \\
\hline
\end{tabular}

\begin{tabular}{|c|c|}
\hline $\mathbf{F}$ \\
\hline $\mathbf{F}$
\end{tabular}

\begin{tabular}{|c|c|}
\hline C \\
\hline C
\end{tabular}

\begin{tabular}{|l|}
$\mathbf{C}$ \\
\hline $\mathbf{C}$ \\
\hline $\mathbf{C}$
\end{tabular}

4.7689706

5.5659995

5.0055824

3.6663493

2.7839235

C 1.4190899

C

0.5583538

C 3.3527423

F 5.2503054

F

2.6114455

$-0.8191461$

\begin{tabular}{|c|}
\hline $\mathbf{C}$ \\
\hline $\mathbf{C}$ \\
\hline $\mathbf{C}$
\end{tabular}

\begin{tabular}{|l|}
\hline $\mathbf{C}$ \\
\hline $\mathbf{C}$ \\
\hline
\end{tabular}

C -1.6

1.1277801

2.5199327

4.5002718

3.1591483

$-1.6641122$

C 0.2823769

C 5.1399702

C 2.3506616

C $\quad-3.0542912$

\begin{tabular}{|r|}
\hline $\mathbf{C}$ \\
\hline $\mathbf{C}$ \\
\hline $\mathbf{F}$
\end{tabular}

\begin{tabular}{|l}
\hline F \\
\hline $\mathbf{C}$ \\
\hline C
\end{tabular}

\begin{tabular}{|c|}
\hline $\mathbf{C}$ \\
\hline $\mathbf{C}$
\end{tabular}

\begin{tabular}{|c|}
\hline $\mathbf{C}$ \\
\hline $\mathbf{C}$ \\
\hline $\mathbf{F}$
\end{tabular}

\begin{tabular}{|r|}
\hline F \\
\hline $\mathbf{C}$ \\
\hline F
\end{tabular}

\begin{tabular}{|l} 
F \\
\hline C
\end{tabular}

C -1.094657

C 4.4218367

F 6.4567718

C 0.9804396

C 3.0052355

C -3.8841521

C -1.9533522

F 5.0751483

C 0.1806022

F 0.3831742

C 2.2311328

C

C

C

C

$\mathbf{F}$

C

2.2311328
-5.2963909
-3.3153847

$-3.3153847$

$-1.2048094$

0.8334747

2.8370199

$-6.0897448$

$-4.1939145$

0.2922708

1.3051086

2.4336728

2.5127216

1.4706623

1.5199402

0.4827922

0.3252644

1.1548541

1.1624696

0.5191399

$-0.6637691$

$-0.6969404$

0.1378902

0.1231808

$-0.5175762$

$-1.6988395$

$-0.9360497$

$-0.9704127$

$-0.4909199$

$-1.6639915$

$-1.9968921$

$-0.8705534$

$-1.0304845$

$-2.0690457$

$-1.5229543$

$-2.7080358$

$-2.975128$

$-2.1074787$

$-0.0170172$

$-3.1290221$

$-1.5041007$

$-2.6682032$

$-2.1730445$

$-3.2024833$

$-4.1349026$

$-2.5316834$

$-3.7242263$

Z

$-0.5541844$

$-0.1217931$

0.5525619

0.7727205

0.3410902

0.5621415

0.1466643

$-0.34343$

4.5431397

4.9689051

0.3782145

$-0.5380049$

$-0.7620234$

4.125922

4.3344797

$-0.0260462$

$-0.9454246$

3.4519784

3.8876758

0.2107643

$-0.7109227$

3.0035543

3.2714871

4.078461

3.1967455

$-0.1888949$

$-1.1130791$

2.3723924

3.636843

4.7124297

2.7561265

0.0486783

$-0.8735192$

3.8277211

2.9456273

2.1179317

$-0.3549543$

$-1.2784121$ 


\begin{tabular}{|c|c|c|c|}
\hline Element symbol & $\mathbf{X}$ & $\mathbf{Y}$ & $\mathbf{Z}$ \\
\hline $\mathrm{C}$ & -1.9910762 & -3.2431752 & 3.3859495 \\
\hline $\mathbf{F}$ & -1.8056729 & -1.1642059 & 4.4621232 \\
\hline $\mathbf{C}$ & 0.0464096 & -4.271362 & 2.5013433 \\
\hline $\mathrm{C}$ & -5.5291968 & -3.6602785 & -1.0295655 \\
\hline $\mathbf{C}$ & -3.3897181 & -3.3151255 & 3.5727765 \\
\hline $\mathrm{C}$ & -1.3381322 & -4.3378883 & 2.6942778 \\
\hline $\mathbf{F}$ & 0.6465532 & -5.2773865 & 1.8610424 \\
\hline $\mathrm{C}$ & -4.1628532 & -4.3763766 & 3.134244 \\
\hline $\mathbf{F}$ & -3.9963143 & -2.3068139 & 4.2052151 \\
\hline $\mathbf{C}$ & -2.1386654 & -5.4133478 & 2.2493919 \\
\hline $\mathbf{C}$ & -5.5806158 & -4.4454848 & 3.3218616 \\
\hline $\mathbf{C}$ & -3.5079212 & -5.4748461 & 2.4432624 \\
\hline $\mathbf{F}$ & -1.5427007 & -6.4228057 & 1.6074506 \\
\hline $\mathrm{C}$ & -6.2978482 & -5.5071895 & 2.8745127 \\
\hline $\mathbf{F}$ & -6.2355843 & -3.4632984 & 3.9441343 \\
\hline $\mathrm{C}$ & -4.3164554 & -6.5674677 & 1.9936099 \\
\hline $\mathrm{C}$ & -5.6574724 & -6.5818275 & 2.201756 \\
\hline $\mathbf{F}$ & -7.616769 & -5.5695765 & 3.0467991 \\
\hline $\mathbf{F}$ & -3.7696544 & -7.6032302 & 1.3527961 \\
\hline $\mathbf{F}$ & -6.408951 & -7.5969524 & 1.7802564 \\
\hline $\mathbf{H}$ & 3.2410787 & 3.3679601 & 1.2848788 \\
\hline $\mathbf{H}$ & 5.6604681 & 3.2282887 & 0.8878797 \\
\hline $\mathbf{H}$ & 6.6357031 & 1.2641677 & -0.285606 \\
\hline $\mathbf{H}$ & 5.1936437 & -0.5642517 & -1.0646602 \\
\hline $\mathbf{H}$ & 0.9937785 & 2.3763897 & 1.0740382 \\
\hline $\mathbf{H}$ & 2.9452924 & -1.5535736 & -1.2735411 \\
\hline $\mathbf{H}$ & 0.7078052 & -2.555722 & -1.4561426 \\
\hline $\mathbf{H}$ & -1.244425 & 1.3753615 & 0.8902259 \\
\hline $\mathbf{H}$ & -1.5283397 & -3.564679 & -1.6248316 \\
\hline $\mathbf{H}$ & -3.479522 & 0.3652817 & 0.7230068 \\
\hline $\mathbf{H}$ & -5.7213927 & -0.6476736 & 0.5591345 \\
\hline $\mathbf{H}$ & -3.7687147 & -4.5788531 & -1.7918272 \\
\hline $\mathbf{H}$ & -6.181084 & -4.4653744 & -1.3458254 \\
\hline $\mathbf{H}$ & -7.156465 & -2.5012874 & -0.1697362 \\
\hline
\end{tabular}




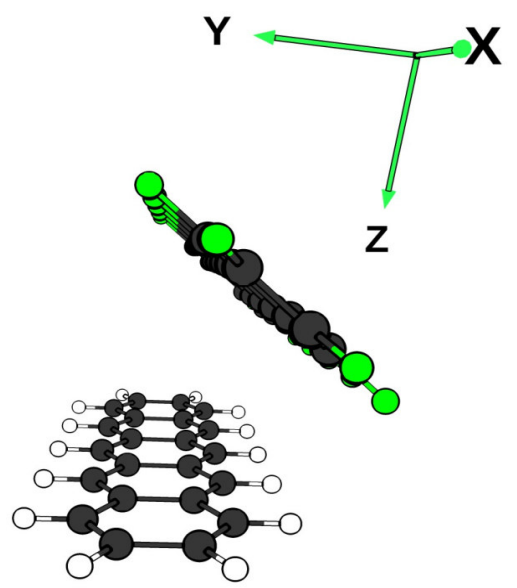

Figure S8. Plot of the cartesian coordinates given in Table S2 for the PEN-PFP herringbone dimer.

Table S2. Cartesian coordinates of a herringbone PEN-PFP dimer in Ångstrom.

\begin{tabular}{|c|c|c|c|}
\hline Element symbol & $\mathbf{X}$ & $\mathbf{Y}$ & $\mathbf{Z}$ \\
\hline $\mathbf{F}$ & 0.3124781 & 5.8146158 & 7.0236325 \\
\hline $\mathbf{F}$ & 14.8681069 & 3.497322 & 5.3074435 \\
\hline $\mathbf{F}$ & 0.6467055 & 3.6605448 & 8.5797022 \\
\hline $\mathbf{F}$ & 14.530754 & 5.6365781 & 3.7317296 \\
\hline $\mathbf{F}$ & 3.0373404 & 2.5003062 & 8.8650174 \\
\hline $\mathbf{F}$ & 12.1293116 & 6.7695154 & 3.4109073 \\
\hline $\mathbf{F}$ & 5.5005165 & 2.4661115 & 8.2733083 \\
\hline $\mathbf{F}$ & 9.661149 & 6.7857194 & 3.980421 \\
\hline $\mathbf{F}$ & 7.24167 & 6.8152406 & 4.5408251 \\
\hline $\mathbf{F}$ & 7.9205831 & 2.4357206 & 7.7120482 \\
\hline $\mathbf{F}$ & 4.8239107 & 6.851935 & 5.1082895 \\
\hline $\mathbf{F}$ & 10.3434321 & 2.4135504 & 7.1630936 \\
\hline $\mathbf{F}$ & 2.3579653 & 6.9014163 & 5.6866198 \\
\hline $\mathbf{F}$ & 12.8184358 & 2.3930976 & 6.6213079 \\
\hline $\mathrm{C}$ & 12.2774385 & 7.2730141 & 10.1163621 \\
\hline C & 2.5040529 & 4.8270708 & 11.9763432 \\
\hline $\mathrm{C}$ & 2.5819492 & 5.8284953 & 6.447571 \\
\hline $\mathrm{C}$ & 12.5906902 & 3.4585845 & 5.8498997 \\
\hline $\mathrm{C}$ & 13.38339 & 6.5431592 & 9.8122246 \\
\hline $\mathrm{C}$ & 1.3954257 & 5.558266 & 12.2674933 \\
\hline $\mathbf{C}$ & 1.5317086 & 5.2906765 & 7.1190333 \\
\hline C & 13.6432673 & 4.0050443 & 5.1899647 \\
\hline C & 13.306024 & 5.1205553 & 9.697171 \\
\hline $\mathrm{C}$ & 1.4712315 & 6.9813204 & 12.3757745 \\
\hline $\mathrm{C}$ & 1.7086422 & 4.1474175 & 7.9433037 \\
\hline $\mathrm{C}$ & 13.465034 & 5.1417298 & 4.3565543 \\
\hline $\mathrm{C}$ & 12.1256477 & 4.4741317 & 9.8923568 \\
\hline C & 2.6524382 & 7.6271944 & 12.1871496 \\
\hline $\mathrm{C}$ & 2.930418 & 3.5713494 & 8.074221 \\
\hline $\mathrm{C}$ & 12.2384797 & 5.7035903 & 4.206037 \\
\hline $\mathrm{C}$ & 10.9321209 & 5.1970658 & 10.216303 \\
\hline
\end{tabular}




\begin{tabular}{|c|c|c|c|}
\hline Element symbol & $\mathbf{X}$ & $\mathbf{Y}$ & $\mathbf{Z}$ \\
\hline C & 3.8487257 & 6.904047 & 11.875286 \\
\hline $\mathbf{C}$ & 4.0747668 & 4.0936577 & 7.3910767 \\
\hline C & 11.0916064 & 5.1718769 & 4.878993 \\
\hline $\mathrm{C}$ & 9.718812 & 4.5644579 & 10.4225407 \\
\hline $\mathrm{C}$ & 5.0632435 & 7.535542 & 11.67528 \\
\hline C & 5.3400942 & 3.541704 & 7.4955352 \\
\hline $\mathbf{C}$ & 9.8232319 & 5.7148145 & 4.761662 \\
\hline $\mathrm{C}$ & 8.5470849 & 5.283451 & 10.7398198 \\
\hline $\mathbf{C}$ & 6.236838 & 6.8165337 & 11.3662424 \\
\hline $\mathbf{C}$ & 6.4715399 & 4.058193 & 6.8262689 \\
\hline $\mathrm{C}$ & 8.6908227 & 5.1939366 & 5.4271607 \\
\hline $\mathrm{C}$ & 7.4665683 & 7.447621 & 11.1606608 \\
\hline $\mathbf{C}$ & 7.318112 & 4.6514821 & 10.9491843 \\
\hline $\mathrm{C}$ & 7.4079399 & 5.7435967 & 5.3175443 \\
\hline $\mathrm{C}$ & 7.7543161 & 3.5085288 & 6.934352 \\
\hline $\mathbf{C}$ & 8.62427 & 6.7290405 & 10.850657 \\
\hline $\mathrm{C}$ & 6.1602341 & 5.3706809 & 11.2581604 \\
\hline $\mathrm{C}$ & 6.2910293 & 5.224522 & 5.9834686 \\
\hline $\mathrm{C}$ & 8.8718315 & 4.0293013 & 6.2722125 \\
\hline $\mathbf{C}$ & 9.8687824 & 7.3590635 & 10.6402064 \\
\hline $\mathbf{C}$ & 4.9156927 & 4.7400921 & 11.4678903 \\
\hline $\mathrm{C}$ & 4.9948625 & 5.7778179 & 5.8831477 \\
\hline $\mathrm{C}$ & 10.1707237 & 3.4850568 & 6.3828432 \\
\hline $\mathrm{C}$ & 11.0100082 & 6.6410056 & 10.3304761 \\
\hline C & 3.7723139 & 5.4594466 & 11.7677257 \\
\hline $\mathrm{C}$ & 3.8940431 & 5.2637094 & 6.5478227 \\
\hline $\mathrm{C}$ & 11.2735071 & 4.0064399 & 5.7285918 \\
\hline $\mathbf{H}$ & 0.4433016 & 5.065662 & 12.4212306 \\
\hline $\mathbf{H}$ & 0.5752641 & 7.5424723 & 12.6107989 \\
\hline $\mathbf{H}$ & 2.7099764 & 8.706465 & 12.2695163 \\
\hline $\mathbf{H}$ & 5.1199879 & 8.6157071 & 11.7570929 \\
\hline $\mathbf{H}$ & 7.5240592 & 8.5275109 & 11.2443056 \\
\hline $\mathbf{H}$ & 9.9274957 & 8.4388236 & 10.726269 \\
\hline $\mathbf{H}$ & 12.3358645 & 8.3518515 & 10.2039019 \\
\hline $\mathbf{H}$ & 14.3351201 & 7.0359464 & 9.6558077 \\
\hline $\mathbf{H}$ & 14.2001074 & 4.5590137 & 9.4554272 \\
\hline $\mathbf{H}$ & 12.0660592 & 3.3957248 & 9.8024677 \\
\hline $\mathbf{H}$ & 9.6605957 & 3.4849301 & 10.3355654 \\
\hline $\mathbf{H}$ & 7.2606028 & 3.5717098 & 10.8660384 \\
\hline $\mathbf{H}$ & 4.858561 & 3.6598577 & 11.3886141 \\
\hline $\mathbf{H}$ & 2.4474818 & 3.747753 & 11.8950985 \\
\hline
\end{tabular}




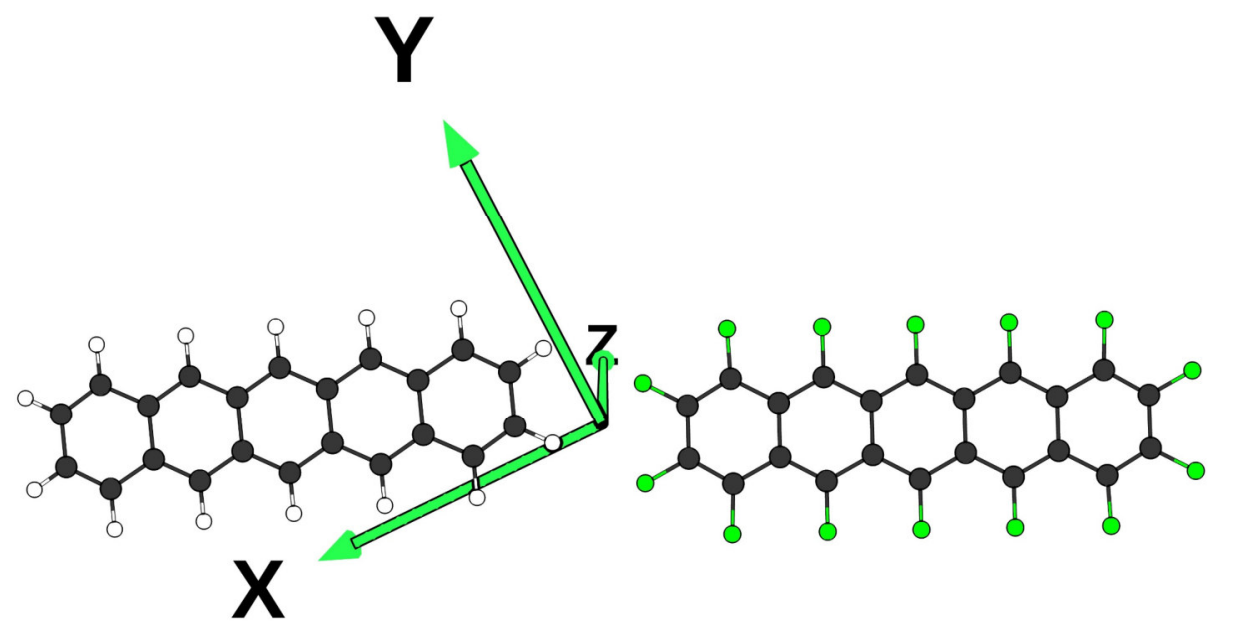

Figure S9. Plot of the cartesian coordinates given in Table S3 for the PEN-PFP end-on dimer.

Table S3. Cartesian coordinates of a end-on PEN-PFP dimer in Ångstrom.

\begin{tabular}{|c|c|c|c|}
\hline Element symbol & X & $\mathbf{Y}$ & $\mathbf{Z}$ \\
\hline C & -2.2291728 & -0.6225538 & 0.186058 \\
\hline $\mathrm{C}$ & -3.575104 & -0.5046278 & 0.3185806 \\
\hline $\mathrm{C}$ & -4.434883 & -1.6414296 & 0.1775352 \\
\hline $\mathrm{C}$ & -5.8121378 & -1.5719259 & 0.3068134 \\
\hline $\mathrm{C}$ & -6.6626669 & -2.6920056 & 0.1677669 \\
\hline $\mathrm{C}$ & 2.3724042 & 3.41063 & 0.087989 \\
\hline C & 1.5214741 & 2.3637594 & -0.07916 \\
\hline $\mathrm{C}$ & 3.7908167 & 3.2317212 & 0.0020489 \\
\hline $\mathbf{F}$ & -1.4288318 & 0.4336274 & 0.3165582 \\
\hline $\mathbf{F}$ & -4.0773256 & 0.7020534 & 0.5843157 \\
\hline $\mathbf{F}$ & -6.3652869 & -0.387045 & 0.5762896 \\
\hline H & 0.4510643 & 2.5130708 & -0.0107407 \\
\hline $\mathrm{C}$ & -1.635273 & -1.8810589 & -0.0991878 \\
\hline $\mathrm{C}$ & -3.8270599 & -2.9298058 & -0.1150786 \\
\hline $\mathrm{C}$ & -8.0554056 & -2.6270445 & 0.2975084 \\
\hline $\mathrm{C}$ & -6.0562748 & -3.9772134 & -0.1245974 \\
\hline $\mathbf{H}$ & 1.9907657 & 4.40486 & 0.2904254 \\
\hline $\mathrm{C}$ & 2.0253106 & 1.0530772 & -0.3455691 \\
\hline C & 4.3022368 & 1.9015858 & -0.2684517 \\
\hline $\mathrm{C}$ & 4.6795597 & 4.278811 & 0.1671509 \\
\hline $\mathrm{C}$ & -2.4020535 & -2.9916281 & -0.2457882 \\
\hline $\mathbf{F}$ & -0.3106397 & -1.9378527 & -0.2207549 \\
\hline $\mathrm{C}$ & -4.6500459 & -4.0351843 & -0.2529104 \\
\hline $\mathrm{C}$ & -8.8918205 & -3.7417982 & 0.1588384 \\
\hline $\mathbf{F}$ & -8.6127672 & -1.4456006 & 0.5660575 \\
\hline $\mathrm{C}$ & -6.8924058 & -5.0916881 & -0.2637868 \\
\hline $\mathbf{H}$ & 1.3270073 & 0.2360833 & -0.4752864 \\
\hline $\mathrm{C}$ & 3.3636419 & 0.8331813 & -0.4366534 \\
\hline C & 5.6686954 & 1.7056743 & -0.3558606 \\
\hline $\mathbf{H}$ & 4.2968062 & 5.2735501 & 0.3693041 \\
\hline C & 6.0752835 & 4.0946505 & 0.08033 \\
\hline
\end{tabular}




\begin{tabular}{|c|c|c|c|}
\hline Element symbol & $\mathbf{X}$ & $\mathbf{Y}$ & $\mathbf{Z}$ \\
\hline $\mathbf{F}$ & -1.7911804 & -4.1460212 & -0.515285 \\
\hline $\mathbf{F}$ & -4.0873992 & -5.2158601 & -0.5209774 \\
\hline $\mathbf{C}$ & -10.2976971 & -3.6845408 & 0.2892181 \\
\hline $\mathrm{C}$ & -8.2853776 & -5.0268182 & -0.1341272 \\
\hline $\mathbf{F}$ & -6.3350076 & -6.2729075 & -0.5332516 \\
\hline $\mathbf{H}$ & 3.7463575 & -0.1606696 & -0.6385984 \\
\hline $\mathbf{H}$ & 6.0505396 & 0.7108039 & -0.5581292 \\
\hline $\mathrm{C}$ & 6.5869634 & 2.7631351 & -0.1901231 \\
\hline $\mathrm{C}$ & 6.9791397 & 5.1473106 & 0.2451187 \\
\hline $\mathrm{C}$ & -11.1207335 & -4.7902719 & 0.151405 \\
\hline $\mathbf{F}$ & -10.8604271 & -2.5042204 & 0.559089 \\
\hline $\mathrm{C}$ & -9.1354306 & -6.1469933 & -0.2727465 \\
\hline $\mathbf{C}$ & 7.9682615 & 2.5729001 & -0.2775682 \\
\hline $\mathbf{H}$ & 6.597017 & 6.1422089 & 0.4469745 \\
\hline $\mathrm{C}$ & 8.3605683 & 4.95706 & 0.1572207 \\
\hline $\mathbf{C}$ & -12.5453206 & -4.7297652 & 0.2845699 \\
\hline $\mathrm{C}$ & -10.512879 & -6.0780943 & -0.1427336 \\
\hline $\mathbf{F}$ & -8.5818791 & -7.3316343 & -0.5427496 \\
\hline $\mathbf{H}$ & 8.3503259 & 1.578268 & -0.4796257 \\
\hline $\mathrm{C}$ & 8.8720532 & 3.6254348 & -0.1130302 \\
\hline $\mathbf{C}$ & 9.2790757 & 6.0147769 & 0.3213259 \\
\hline $\mathrm{C}$ & -13.3125326 & -5.8409686 & 0.1409157 \\
\hline $\mathbf{F}$ & -13.1563277 & -3.5751299 & 0.5542879 \\
\hline C & -11.3724358 & -7.2148797 & -0.2834051 \\
\hline $\mathbf{C}$ & 10.2675323 & 3.4411332 & -0.2008966 \\
\hline H & 8.8974278 & 7.0099494 & 0.5233367 \\
\hline $\mathrm{C}$ & 10.6454894 & 5.8186854 & 0.2321052 \\
\hline $\mathbf{F}$ & -14.6355418 & -5.7842084 & 0.2647147 \\
\hline $\mathrm{C}$ & -12.7185841 & -7.0993201 & -0.1466803 \\
\hline $\mathbf{F}$ & -10.8696407 & -8.4206389 & -0.5531707 \\
\hline $\mathbf{H}$ & 10.6500735 & 2.4464342 & -0.4026212 \\
\hline $\mathrm{C}$ & 11.1563552 & 4.4882858 & -0.0377713 \\
\hline $\mathrm{C}$ & 11.5844695 & 6.8872657 & 0.3973523 \\
\hline $\mathbf{F}$ & -13.5164407 & -8.155294 & -0.2774099 \\
\hline $\mathbf{C}$ & 12.5744572 & 4.3090743 & -0.1255043 \\
\hline $\mathbf{H}$ & 11.2023313 & 7.8814313 & 0.5989669 \\
\hline $\mathrm{C}$ & 12.9226545 & 6.6666472 & 0.3050535 \\
\hline $\mathbf{H}$ & 12.9557899 & 3.3147196 & -0.3272728 \\
\hline $\mathrm{C}$ & 13.4260314 & 5.3557378 & 0.0393412 \\
\hline $\mathbf{H}$ & 13.6198653 & 7.4855697 & 0.4325865 \\
\hline $\mathbf{H}$ & 14.4961445 & 5.203531 & -0.0296488 \\
\hline
\end{tabular}




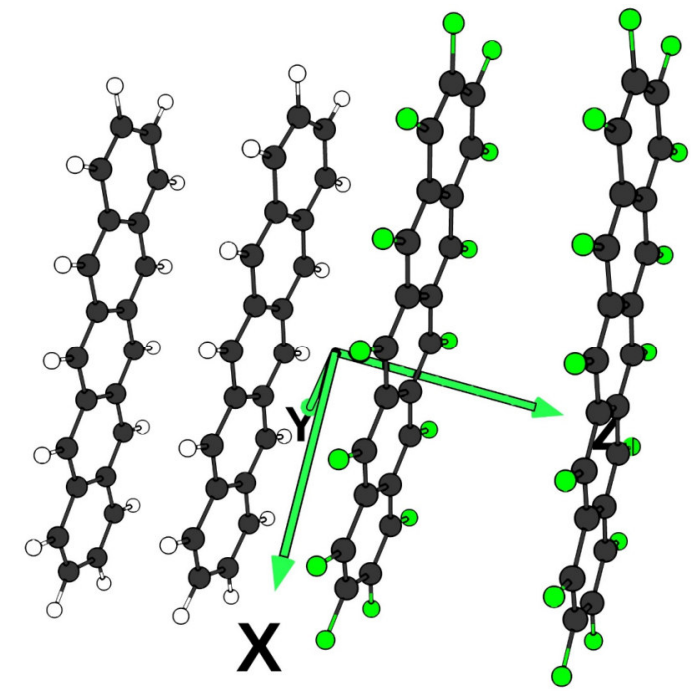

Figure S10. Plot of the cartesian coordinates given in Table S4 for the PEN-PFP tetramer.

Table S4. Cartesian coordinates of a slip $\pi$-stacked PEN-PFP tetramer in Ångstrom.

\begin{tabular}{|c|c|c|c|}
\hline Element symbol & $\mathbf{X}$ & $\mathbf{Y}$ & $\mathbf{Z}$ \\
\hline $\mathrm{C}$ & 4.7646315 & 0.3858889 & -0.7198969 \\
\hline C & 5.5604497 & 1.4097624 & -0.3133725 \\
\hline $\mathrm{C}$ & 5.002983 & 2.5405818 & 0.3583235 \\
\hline $\mathrm{C}$ & 3.6673124 & 2.612287 & 0.6002749 \\
\hline $\mathrm{C}$ & 2.7856114 & 1.5602422 & 0.1933834 \\
\hline $\mathrm{C}$ & 1.4232015 & 1.6058552 & 0.429487 \\
\hline $\mathrm{C}$ & 0.5621236 & 0.5614752 & 0.0347538 \\
\hline $\mathrm{C}$ & 3.3518103 & 0.4108706 & -0.486202 \\
\hline $\mathbf{F}$ & 5.2354453 & 1.3070622 & 4.3066832 \\
\hline $\mathbf{F}$ & 2.6064744 & 1.2715344 & 4.7880742 \\
\hline $\mathrm{C}$ & -0.8123776 & 0.5928735 & 0.2846278 \\
\hline $\mathrm{C}$ & 1.1287713 & -0.5884262 & -0.645788 \\
\hline $\mathrm{C}$ & 2.518487 & -0.6186609 & -0.8844831 \\
\hline $\mathbf{C}$ & 4.4978987 & 0.2690114 & 3.9229928 \\
\hline $\mathrm{C}$ & 3.1614897 & 0.2329523 & 4.1586833 \\
\hline $\mathrm{C}$ & -1.6569963 & -0.4528959 & -0.0953059 \\
\hline $\mathrm{C}$ & 0.2824338 & -1.628702 & -1.0365212 \\
\hline $\mathrm{C}$ & 5.1448427 & -0.8043586 & 3.2554583 \\
\hline $\mathrm{C}$ & 2.3648963 & -0.8815968 & 3.7449832 \\
\hline $\mathrm{C}$ & -3.0413826 & -0.4397873 & 0.1750062 \\
\hline $\mathrm{C}$ & -1.0911173 & -1.5984207 & -0.7837721 \\
\hline C & 4.4371604 & -1.88487 & 2.838151 \\
\hline $\mathbf{F}$ & 6.4557573 & -0.7180853 & 3.0485841 \\
\hline $\mathrm{C}$ & 0.9996903 & -0.962655 & 3.9616533 \\
\hline $\mathrm{C}$ & 3.0260642 & -1.9782225 & 3.0585501 \\
\hline $\mathrm{C}$ & -3.8676736 & -1.4862933 & -0.1927744 \\
\hline $\mathrm{C}$ & -1.9484885 & -2.6501022 & -1.1660201 \\
\hline $\mathbf{F}$ & 5.0946207 & -2.8612053 & 2.2103079 \\
\hline $\mathrm{C}$ & 0.210824 & -2.0580528 & 3.5483164 \\
\hline
\end{tabular}




\begin{tabular}{|c|c|c|c|}
\hline $\mathbf{F}$ & 0.3965117 & 0.0478071 & 4.5951938 \\
\hline C & 2.262614 & -3.0570489 & 2.645699 \\
\hline C & -5.2707981 & -1.4917122 & 0.0951761 \\
\hline $\mathbf{C}$ & -3.3036173 & -2.625296 & -0.8907863 \\
\hline C & -1.1695254 & -2.1446381 & 3.7643773 \\
\hline C & 0.8695392 & -3.1499355 & 2.8586209 \\
\hline $\mathbf{F}$ & 2.8740245 & -4.0610753 & 2.0128292 \\
\hline C & -6.0591378 & -2.5366143 & -0.2728534 \\
\hline C & -4.1777133 & -3.6955037 & -1.2647724 \\
\hline C & -1.9455974 & -3.2311298 & 3.3469598 \\
\hline $\mathbf{F}$ & -1.7763361 & -1.1397373 & 4.3999856 \\
\hline C & 0.0927275 & -4.2365019 & 2.4394746 \\
\hline C & -5.50351 & -3.6564176 & -0.966013 \\
\hline C & -3.3398884 & -3.3214521 & 3.5538544 \\
\hline C & -1.2873198 & -4.3226609 & 2.6562292 \\
\hline $\mathbf{F}$ & 0.6976557 & -5.2376398 & 1.7993664 \\
\hline C & -4.1035798 & -4.3970578 & 3.1355991 \\
\hline $\mathbf{F}$ & -3.9526099 & -2.3179772 & 4.1889334 \\
\hline C & -2.0776947 & -5.4153597 & 2.2348567 \\
\hline C & -5.5165794 & -4.4855213 & 3.3464077 \\
\hline C & -3.4434643 & -5.4940034 & 2.4478053 \\
\hline $\mathbf{F}$ & -1.4763517 & -6.4207815 & 1.5956726 \\
\hline C & -6.2233169 & -5.5654835 & 2.9272357 \\
\hline $\mathbf{F}$ & -6.1768665 & -3.5080285 & 3.9715338 \\
\hline C & -4.2417415 & -6.6046981 & 2.0237402 \\
\hline C & -5.5787865 & -6.6385772 & 2.2559414 \\
\hline $\mathbf{F}$ & -7.5363254 & -5.6500407 & 3.1342213 \\
\hline $\mathbf{F}$ & -3.6894511 & -7.6362839 & 1.3849508 \\
\hline $\mathbf{F}$ & -6.3214346 & -7.6707412 & 1.8648738 \\
\hline $\mathbf{H}$ & 3.2432805 & 3.4721855 & 1.1057451 \\
\hline $\mathbf{H}$ & 5.6572151 & 3.3463625 & 0.667353 \\
\hline $\mathbf{H}$ & 6.6255997 & 1.3810901 & -0.5062417 \\
\hline $\mathbf{H}$ & 5.1863335 & -0.4682729 & -1.2370824 \\
\hline $\mathbf{H}$ & 0.999319 & 2.4666079 & 0.9356432 \\
\hline H & 2.9400665 & -1.4743251 & -1.4011455 \\
\hline $\mathbf{H}$ & 0.704209 & -2.4848474 & -1.5519205 \\
\hline $\mathbf{H}$ & -1.2350063 & 1.4510531 & 0.7959349 \\
\hline $\mathbf{H}$ & -1.5268161 & -3.501452 & -1.6895234 \\
\hline $\mathbf{H}$ & -3.4638042 & 0.414493 & 0.6932489 \\
\hline $\mathbf{H}$ & -5.6926113 & -0.6408452 & 0.6179391 \\
\hline $\mathbf{H}$ & -3.7551419 & -4.5413002 & -1.7947263 \\
\hline $\mathbf{H}$ & -6.1514507 & -4.4742038 & -1.2575954 \\
\hline $\mathbf{H}$ & -7.1183597 & -2.5267243 & -0.0462159 \\
\hline C & -4.6688346 & -2.179333 & -5.3482529 \\
\hline C & -3.3162351 & -2.1972191 & -5.4808535 \\
\hline C & -5.3306018 & -1.034661 & -4.806836 \\
\hline C & -4.6178618 & 0.0557335 & -4.4196189 \\
\hline $\mathbf{C}$ & -3.1917514 & 0.0893009 & -4.5427582 \\
\hline C & -2.5196127 & -1.0740426 & -5.0876854 \\
\hline
\end{tabular}




\begin{tabular}{|c|c|c|c|}
\hline C & -2.4433837 & 1.1879764 & -4.1618416 \\
\hline C & -1.1420908 & -1.0652568 & -5.2096736 \\
\hline C & -0.3650273 & 0.0446196 & -4.8189244 \\
\hline C & -1.0386259 & 1.212181 & -4.2813079 \\
\hline $\mathrm{C}$ & -0.2757641 & 2.3170697 & -3.8962972 \\
\hline C & 1.0275169 & 0.0555495 & -4.9251819 \\
\hline C & 1.7915205 & 1.1556788 & -4.5287922 \\
\hline $\mathrm{C}$ & 1.1170792 & 2.3278282 & -4.0021764 \\
\hline C & 1.8945934 & 3.4356782 & -3.6066944 \\
\hline $\mathrm{C}$ & 3.1990165 & 1.1651115 & -4.6119392 \\
\hline C & 3.9493883 & 2.2547426 & -4.2093802 \\
\hline C & 3.2747042 & 3.4302812 & -3.6943278 \\
\hline C & 4.0718361 & 4.5453781 & -3.2812589 \\
\hline C & 5.428156 & 4.5066891 & -3.3595597 \\
\hline C & 6.0934869 & 3.3456576 & -3.8609903 \\
\hline $\mathrm{C}$ & 5.3796971 & 2.2640193 & -4.2705209 \\
\hline $\mathbf{H}$ & 5.8836237 & 1.3808398 & -4.6461188 \\
\hline $\mathbf{H}$ & 7.1755523 & 3.3343488 & -3.9092033 \\
\hline $\mathbf{H}$ & 6.0167524 & 5.3569078 & -3.0373184 \\
\hline $\mathbf{H}$ & 3.5664828 & 5.4245348 & -2.898114 \\
\hline $\mathbf{H}$ & 1.3906208 & 4.3127114 & -3.2147115 \\
\hline $\mathbf{H}$ & 3.7034861 & 0.2844679 & -4.9953623 \\
\hline $\mathbf{H}$ & 1.5312155 & -0.8195082 & -5.3218829 \\
\hline $\mathbf{H}$ & -0.7794897 & 3.1905404 & -3.4966145 \\
\hline $\mathbf{H}$ & -2.9466636 & 2.0586158 & -3.7550149 \\
\hline $\mathbf{H}$ & -0.639301 & -1.9359577 & -5.617529 \\
\hline $\mathbf{H}$ & -2.8138116 & -3.0665462 & -5.8898378 \\
\hline $\mathbf{H}$ & -5.1182091 & 0.9230973 & -4.0046549 \\
\hline $\mathbf{H}$ & -6.4088412 & -1.0450979 & -4.7055512 \\
\hline $\mathbf{H}$ & -5.2575567 & -3.0367218 & -5.6507921 \\
\hline $\mathbf{C}$ & -6.1057618 & -7.827138 & 6.0155501 \\
\hline $\mathbf{F}$ & -6.8636622 & -8.7232709 & 5.3916873 \\
\hline $\mathbf{F}$ & -8.0582104 & -6.9671299 & 7.0246803 \\
\hline $\mathbf{F}$ & -6.6585862 & -5.0874417 & 8.3139516 \\
\hline C & -6.0129409 & -5.9412792 & 7.5198188 \\
\hline C & -6.7398601 & -6.8893409 & 6.8740691 \\
\hline C & -4.5927855 & -5.8561782 & 7.3547869 \\
\hline C & -3.9422621 & -6.8266909 & 6.4899496 \\
\hline $\mathrm{C}$ & -4.7607553 & $\begin{array}{l}-7.799878 \\
\end{array}$ & 5.8329651 \\
\hline $\mathbf{F}$ & -4.2216242 & -8.6997406 & 5.0079362 \\
\hline C & -2.5672168 & -6.7642071 & 6.3374188 \\
\hline C & -1.7592399 & -5.7967364 & 6.9741761 \\
\hline $\mathbf{F}$ & -1.9754342 & -7.6576244 & 5.5398943 \\
\hline C & -3.8134854 & -4.8908921 & 7.9706431 \\
\hline $\mathbf{F}$ & -4.4137476 & -3.9972248 & 8.7584239 \\
\hline C & -2.4124923 & -4.8045111 & 7.806179 \\
\hline C & -1.6244046 & -3.8110855 & 8.3994307 \\
\hline $\mathbf{F}$ & -2.2260879 & -2.8866061 & 9.1470729 \\
\hline C & -0.2340462 & -3.7429691 & 8.246608 \\
\hline
\end{tabular}




\begin{tabular}{|lccc|}
\hline $\mathbf{C}$ & 0.4246583 & -4.7659643 & 7.4572561 \\
\hline $\mathbf{C}$ & -0.3659569 & -5.7461664 & 6.8463258 \\
\hline $\mathbf{F}$ & 0.2376647 & -6.678884 & 6.1075515 \\
\hline $\mathbf{C}$ & 1.8324419 & -4.7172017 & 7.348146 \\
\hline $\mathbf{F}$ & 2.4461455 & -5.6715834 & 6.6440887 \\
\hline $\mathbf{C}$ & 2.6069806 & -3.7302927 & 7.9345034 \\
\hline $\mathbf{C}$ & 1.943409 & -2.6846717 & 8.6954787 \\
\hline $\mathbf{C}$ & 0.5649468 & -2.7308646 & 8.8233901 \\
\hline $\mathbf{F}$ & -0.0400718 & -1.7716553 & 9.5260083 \\
\hline $\mathbf{C}$ & 2.7508467 & -1.6567381 & 9.2796688 \\
\hline $\mathbf{F}$ & 2.193335 & -0.6686465 & 9.9798535 \\
\hline $\mathbf{F}$ & 4.1015385 & -1.6595431 & 9.139735 \\
\hline $\mathbf{C}$ & 4.8519069 & -0.7061195 & 9.683903 \\
\hline $\mathbf{F}$ & 4.7511176 & -2.6881401 & 8.405948 \\
\hline $\mathbf{F}$ & 6.0765591 & -2.6467407 & 8.3047622 \\
\hline $\mathbf{C}$ & 4.6967632 & -4.6276322 & 7.1547803 \\
\hline
\end{tabular}

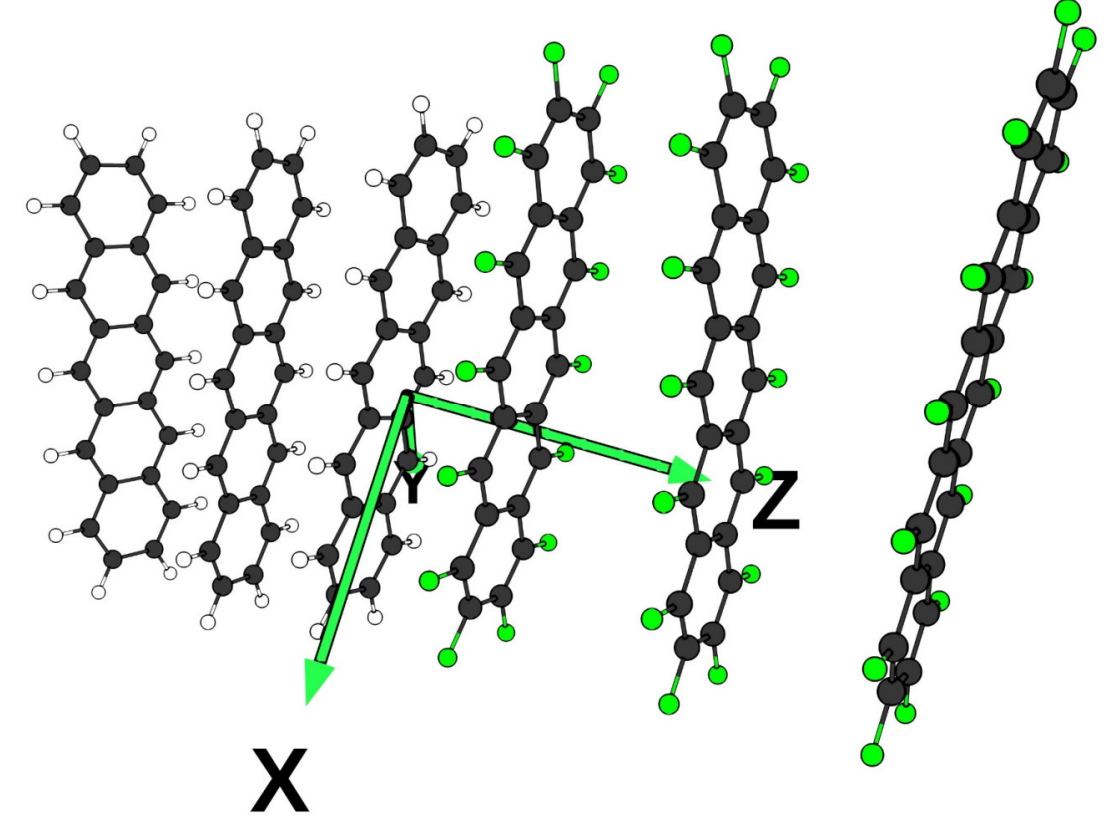

Figure S11. Plot of the cartesian coordinates given in Table S5 for the PEN-PFP hexamer. 
Table S5. Cartesian coordinates of a slip $\pi$-stacked PEN-PFP hexamer in Ångstrom.

\begin{tabular}{|c|c|c|c|}
\hline Element symbol & $\mathbf{X}$ & $\mathbf{Y}$ & $\mathbf{Z}$ \\
\hline $\mathrm{C}$ & 4.7543521 & 0.3333038 & -0.7171283 \\
\hline $\mathbf{C}$ & 5.5498127 & 1.3490739 & -0.2904477 \\
\hline $\mathbf{C}$ & 4.9894546 & 2.4760692 & 0.3849493 \\
\hline $\mathbf{C}$ & 3.6517542 & 2.5497444 & 0.6145979 \\
\hline $\mathbf{C}$ & 2.7711750 & 1.5031256 & 0.1919196 \\
\hline $\mathbf{C}$ & 1.4077001 & 1.5475716 & 0.4221166 \\
\hline $\mathbf{C}$ & 0.5479990 & 0.5067066 & 0.0150017 \\
\hline $\mathbf{C}$ & 3.3395743 & 0.3603589 & -0.4964417 \\
\hline $\mathbf{F}$ & 5.2245480 & 1.2258168 & 4.3407542 \\
\hline $\mathbf{F}$ & 2.5927063 & 1.1927026 & 4.8080551 \\
\hline $\mathbf{C}$ & -0.8278391 & 0.5357308 & 0.2581904 \\
\hline $\mathbf{C}$ & 1.1172259 & -0.6364578 & -0.6741479 \\
\hline $\mathbf{C}$ & 2.5072139 & -0.6634242 & -0.9104673 \\
\hline $\mathbf{C}$ & 4.4859771 & 0.1946847 & 3.9411202 \\
\hline $\mathbf{C}$ & 3.1484049 & 0.1598830 & 4.1699810 \\
\hline $\mathbf{C}$ & -1.6709901 & -0.5065225 & -0.1356041 \\
\hline $\mathbf{C}$ & 0.2734653 & -1.6748693 & -1.0743282 \\
\hline $\mathbf{C}$ & 5.1328113 & -0.8732042 & 3.2648259 \\
\hline $\mathbf{C}$ & 2.3513207 & -0.9484558 & 3.7414344 \\
\hline $\mathbf{C}$ & -3.0582304 & -0.4927427 & 0.1201845 \\
\hline $\mathbf{C}$ & -1.1010221 & -1.6478852 & -0.8271144 \\
\hline $\mathbf{C}$ & 4.4245227 & -1.9482669 & 2.8343375 \\
\hline $\mathbf{F}$ & 6.4446517 & -0.7879909 & 3.0643928 \\
\hline $\mathbf{C}$ & 0.9849340 & -1.0271716 & 3.9502905 \\
\hline C & 3.0122516 & -2.0405085 & 3.0478012 \\
\hline C & -3.8831073 & -1.5343369 & -0.2650649 \\
\hline C & -1.9551097 & -2.6985835 & -1.2197402 \\
\hline $\mathbf{F}$ & 5.0826012 & -2.9206314 & 2.2013984 \\
\hline $\mathrm{C}$ & 0.1955202 & -2.1167452 & 3.5241195 \\
\hline $\mathbf{F}$ & 0.3805805 & -0.0198102 & 4.5875426 \\
\hline C & 2.2477151 & -3.1137247 & 2.6218293 \\
\hline $\mathrm{C}$ & -5.2913561 & -1.5328021 & -0.0022613 \\
\hline C & -3.3136163 & -2.6723778 & -0.9605909 \\
\hline C & -1.1860710 & -2.1990277 & 3.7317735 \\
\hline $\mathbf{C}$ & 0.8540013 & -3.2062462 & 2.8307762 \\
\hline $\mathbf{F}$ & 2.8583516 & -4.1122559 & 1.9798798 \\
\hline C & -6.0801570 & -2.5697431 & -0.3913042 \\
\hline $\mathbf{C}$ & -4.1872746 & -3.7378619 & -1.3503375 \\
\hline $\mathrm{C}$ & -1.9630296 & -3.2810691 & 3.3059343 \\
\hline $\mathbf{F}$ & -1.7939377 & -1.1936725 & 4.3655173 \\
\hline C & 0.0760949 & -4.2896592 & 2.4048608 \\
\hline $\mathbf{C}$ & -5.5184179 & -3.6912239 & -1.0765150 \\
\hline C & -3.3589169 & -3.3640879 & 3.5019711 \\
\hline $\mathrm{C}$ & -1.3049846 & -4.3729892 & 2.6157372 \\
\hline $\mathbf{F}$ & 0.6801435 & -5.2897366 & 1.7626581 \\
\hline $\mathbf{C}$ & -4.1248806 & -4.4332971 & 3.0728506 \\
\hline
\end{tabular}




\begin{tabular}{|c|c|c|c|}
\hline $\mathbf{F}$ & -3.9709304 & -2.3595449 & 4.1359209 \\
\hline $\mathbf{C}$ & -2.0976773 & -5.4610688 & 2.1857519 \\
\hline $\mathrm{C}$ & -5.5404290 & -4.5110689 & 3.2676934 \\
\hline $\mathbf{C}$ & -3.4657143 & -5.5316749 & 2.3868489 \\
\hline $\mathbf{F}$ & -1.4964645 & -6.4688269 & 1.5506962 \\
\hline $\mathbf{C}$ & -6.2524698 & -5.5790591 & 2.8282818 \\
\hline $\mathbf{F}$ & -6.1977600 & -3.5345259 & 3.8971555 \\
\hline $\mathrm{C}$ & -4.2685951 & -6.6333503 & 1.9475340 \\
\hline $\mathbf{C}$ & -5.6098605 & -6.6524744 & 2.1561564 \\
\hline $\mathbf{F}$ & -7.5693794 & -5.6505774 & 3.0121804 \\
\hline $\mathbf{F}$ & -3.7169313 & -7.6639029 & 1.3072442 \\
\hline $\mathbf{F}$ & -6.3598613 & -7.6668922 & 1.7344788 \\
\hline $\mathbf{H}$ & 3.2251161 & 3.4073708 & 1.1217056 \\
\hline $\mathbf{H}$ & 5.6429304 & 3.2780523 & 0.7054970 \\
\hline $\mathbf{H}$ & 6.6173795 & 1.3157241 & -0.4688373 \\
\hline $\mathbf{H}$ & 5.1785539 & -0.5165874 & -1.2391303 \\
\hline $\mathbf{H}$ & 0.9825252 & 2.4037165 & 0.9350837 \\
\hline $\mathbf{H}$ & 2.9300597 & -1.5135825 & -1.4351033 \\
\hline $\mathbf{H}$ & 0.6962396 & -2.5240273 & -1.5998508 \\
\hline $\mathbf{H}$ & -1.2525593 & 1.3897433 & 0.7747517 \\
\hline $\mathbf{H}$ & -1.5277821 & -3.5494463 & -1.7397794 \\
\hline $\mathbf{H}$ & -3.4844276 & 0.3588047 & 0.6400507 \\
\hline $\mathbf{H}$ & -5.7176405 & -0.6821029 & 0.5173269 \\
\hline $\mathbf{H}$ & -3.7619039 & -4.5872654 & -1.8723367 \\
\hline $\mathbf{H}$ & -6.1668748 & -4.5037911 & -1.3821371 \\
\hline $\mathbf{H}$ & -7.1441562 & -2.5507723 & -0.1870212 \\
\hline $\mathrm{C}$ & -4.7130796 & -2.1629818 & -5.5127973 \\
\hline C & -3.3577723 & -2.1923213 & -5.6106771 \\
\hline $\mathbf{C}$ & -5.3800673 & -1.0032853 & -5.0116391 \\
\hline $\mathbf{C}$ & -4.6694663 & 0.0884357 & -4.6241900 \\
\hline C & -3.2402357 & 0.1079293 & -4.7065383 \\
\hline C & -2.5633574 & -1.0672914 & -5.2182427 \\
\hline $\mathrm{C}$ & -2.4927643 & 1.2028892 & -4.3135416 \\
\hline $\mathbf{C}$ & -1.1832770 & -1.0696220 & -5.3064131 \\
\hline C & -0.4071426 & 0.0360260 & -4.9019390 \\
\hline $\mathbf{C}$ & -1.0846381 & 1.2106511 & -4.3854716 \\
\hline C & -0.3220422 & 2.3037714 & -3.9679954 \\
\hline C & 0.9885081 & 0.0333173 & -4.9659652 \\
\hline $\mathbf{C}$ & 1.7515280 & 1.1234181 & -4.5402664 \\
\hline $\mathbf{C}$ & 1.0735387 & 2.2982125 & -4.0249053 \\
\hline C & 1.8492895 & 3.3940272 & -3.5952000 \\
\hline $\mathbf{C}$ & 3.1609731 & 1.1235348 & -4.5890914 \\
\hline C & 3.9089335 & 2.2057250 & -4.1627278 \\
\hline C & 3.2307318 & 3.3795906 & -3.6494776 \\
\hline $\mathbf{C}$ & 4.0245294 & 4.4850382 & -3.2068054 \\
\hline $\mathbf{C}$ & 5.3813337 & 4.4433164 & -3.2646069 \\
\hline C & 6.0502778 & 3.2876388 & -3.7726193 \\
\hline $\mathrm{C}$ & 5.3399246 & 2.2118984 & -4.2030025 \\
\hline $\mathbf{H}$ & 5.8467376 & 1.3329018 & -4.5848087 \\
\hline
\end{tabular}




\begin{tabular}{|llll|}
\hline $\mathbf{H}$ & 7.1327748 & 3.2762056 & -3.8106029 \\
\hline $\mathbf{H}$ & 5.9674363 & 5.2881401 & -2.9236762 \\
\hline $\mathbf{H}$ & 3.5133259 & 5.3591824 & -2.8195514 \\
\hline $\mathbf{H}$ & 1.3412567 & 4.2707303 & -3.2068758 \\
\hline $\mathbf{H}$ & 3.6684969 & 0.2437888 & -4.9709443 \\
\hline $\mathbf{H}$ & 1.4954955 & -0.8448981 & -5.3520168 \\
\hline $\mathbf{H}$ & -0.8277525 & 3.1804516 & -3.5775629 \\
\hline $\mathbf{H}$ & -2.9995419 & 2.0823514 & -3.9302069 \\
\hline $\mathbf{H}$ & -0.6771617 & -1.9475994 & -5.6941050 \\
\hline $\mathbf{H}$ & -2.8512211 & -3.0723280 & -5.9907809 \\
\hline $\mathbf{H}$ & -5.1739824 & 0.9666646 & -4.2376089 \\
\hline $\mathbf{H}$ & -6.4608122 & -1.0051596 & -4.9373836 \\
\hline $\mathbf{C}$ & -5.3002361 & -3.0226411 & -5.8122541 \\
\hline $\mathbf{F}$ & -6.0746339 & -7.7783216 & 5.8463807 \\
\hline $\mathbf{F}$ & -6.8333922 & -8.6743464 & 5.2241733 \\
\hline $\mathbf{F}$ & -8.0333365 & -6.8636468 & 6.7934592 \\
\hline $\mathbf{C}$ & -6.6287148 & -4.9875663 & 8.0834080 \\
\hline $\mathbf{C}$ & -5.9810387 & -5.8690767 & 7.3227306 \\
\hline $\mathbf{C}$ & -6.7105844 & -6.8154560 & 6.6760758 \\
\hline $\mathbf{C}$ & -4.5545241 & -5.8205699 & 7.2003495 \\
\hline $\mathbf{C}$ & -3.9048467 & -6.8147287 & 6.3625597 \\
\hline $\mathbf{F}$ & -4.7257345 & -7.7767464 & 5.6929609 \\
\hline $\mathbf{C}$ & -4.1826211 & -8.6959319 & 4.8928929 \\
\hline $\mathbf{C}$ & -2.5257970 & -6.7830856 & 6.2426033 \\
\hline $\mathbf{F}$ & -1.7123730 & -5.8346409 & 6.8996487 \\
\hline $\mathbf{C}$ & -1.9336175 & -7.6775465 & 5.4480548 \\
\hline $\mathbf{F}$ & -3.7693304 & -4.8650209 & 7.8256312 \\
\hline $\mathbf{C}$ & -4.3697233 & -3.9447846 & 8.5812296 \\
\hline $\mathbf{C}$ & -2.3615423 & -4.8181472 & 7.7044650 \\
\hline $\mathbf{F}$ & -1.5645876 & -3.8326957 & 8.3008163 \\
\hline $\mathbf{C}$ & -2.1614065 & -2.8869138 & 9.0243058 \\
\hline $\mathbf{C}$ & -0.1697463 & -3.7941023 & 8.1720628 \\
\hline $\mathbf{C}$ & 0.4811269 & -4.8388163 & 7.4051181 \\
\hline $\mathbf{F}$ & -0.3166884 & -5.8136968 & 6.7961601 \\
\hline $\mathbf{C}$ & 0.2823568 & -6.7703121 & 6.0865521 \\
\hline $\mathbf{F}$ & 1.8894561 & -4.8095250 & 7.3004665 \\
\hline $\mathbf{C}$ & 2.4948014 & -5.7848304 & 6.6199470 \\
\hline $\mathbf{C}$ & 2.6719093 & -3.8137493 & 7.8598207 \\
\hline $\mathbf{C}$ & 2.0183408 & -2.7480920 & 8.6007748 \\
\hline $\mathbf{F}$ & 0.6396924 & -2.7827639 & 8.7365477 \\
\hline $\mathbf{C}$ & 0.0439538 & -1.8080305 & 9.4244315 \\
\hline $\mathbf{F}$ & 2.8351737 & -1.7108768 & 9.1546497 \\
\hline $\mathbf{C}$ & 2.2881215 & -0.7046552 & 9.8359511 \\
\hline $\mathbf{F}$ & 4.1849764 & -1.7229059 & 9.0020764 \\
\hline $\mathbf{C}$ & 4.9439323 & -0.7606823 & 9.5161174 \\
\hline $\mathbf{F}$ & 4.8241621 & -2.7698878 & 8.2850697 \\
\hline $\mathbf{F}$ & 6.1475635 & -2.7344544 & 8.1671215 \\
\hline $\mathbf{C}$ & 4.7493028 & -4.7360209 & 7.0780545 \\
\hline & 4.0977994 & -3.7778031 & \\
\hline & & & \\
\hline
\end{tabular}




\begin{tabular}{|c|c|c|c|}
\hline $\mathbf{H}$ & -6.0081404 & 0.7663183 & -10.1262652 \\
\hline $\mathbf{H}$ & -4.7056422 & -0.6911321 & -11.6655162 \\
\hline $\mathbf{C}$ & -4.1886371 & 0.0676665 & -11.0907940 \\
\hline $\mathbf{C}$ & -4.9370995 & 0.9062146 & -10.2082364 \\
\hline $\mathbf{H}$ & -4.8817541 & 2.5020376 & -8.8037182 \\
\hline $\mathrm{C}$ & -4.3151324 & 1.8675126 & -9.4756798 \\
\hline C & -2.9001755 & 2.0680413 & -9.5672632 \\
\hline $\mathbf{C}$ & -2.1401750 & 1.2138735 & -10.4594978 \\
\hline $\mathbf{C}$ & -2.8428163 & 0.2162708 & -11.2093585 \\
\hline $\mathbf{H}$ & -2.2742606 & -0.4202136 & -11.8778668 \\
\hline $\mathbf{H}$ & -0.2029966 & 0.7459168 & -11.2267695 \\
\hline $\mathbf{C}$ & -0.7717675 & 1.3862124 & -10.5605696 \\
\hline C & -2.2434567 & 3.0445390 & -8.8408224 \\
\hline $\mathbf{H}$ & -2.8119193 & 3.6807962 & -8.1708324 \\
\hline $\mathbf{C}$ & -0.8501799 & 3.2391086 & -8.9406526 \\
\hline $\mathbf{C}$ & -0.0883352 & 2.3772864 & -9.8256658 \\
\hline $\mathbf{C}$ & 1.2932802 & 2.5593175 & -9.9233295 \\
\hline $\mathbf{H}$ & 1.8635219 & 1.9149049 & -10.5838337 \\
\hline $\mathbf{C}$ & 1.9623856 & 3.5492619 & -9.1992798 \\
\hline $\mathbf{C}$ & 1.1998478 & 4.4137580 & -8.3170541 \\
\hline $\mathbf{C}$ & -0.1812581 & 4.2291738 & -8.2167859 \\
\hline $\mathbf{H}$ & -0.7535774 & 4.8723633 & -7.5571688 \\
\hline C & 1.8821833 & 5.4118556 & -7.5912324 \\
\hline $\mathbf{H}$ & 1.3124609 & 6.0558438 & -6.9297150 \\
\hline C & 3.2497788 & 5.5882293 & -7.6970077 \\
\hline C & 4.0114139 & 4.7253050 & -8.5795269 \\
\hline $\mathbf{C}$ & 3.3562107 & 3.7410450 & -9.2966266 \\
\hline $\mathbf{H}$ & 3.9265498 & 3.0949217 & -9.9556521 \\
\hline $\mathbf{C}$ & 3.9512539 & 6.5972852 & -6.9624747 \\
\hline $\mathbf{H}$ & 3.3839461 & 7.2425775 & -6.3015081 \\
\hline $\mathbf{C}$ & 5.2967395 & 6.7461407 & -7.0832839 \\
\hline $\mathbf{H}$ & 5.8129088 & 7.5102092 & -6.5149993 \\
\hline $\mathbf{H}$ & 7.1179892 & 6.0379905 & -8.0375979 \\
\hline $\mathrm{C}$ & 6.0468509 & 5.8981289 & -7.9549265 \\
\hline $\mathbf{C}$ & 5.4262100 & 4.9265087 & -8.6746671 \\
\hline $\mathbf{H}$ & 5.9937664 & 4.2831168 & -9.3375202 \\
\hline C & -4.3708139 & -11.2015346 & 10.7232983 \\
\hline $\mathbf{C}$ & -5.7008402 & -11.2901021 & 10.9830743 \\
\hline $\mathbf{F}$ & -6.4016864 & -12.3611136 & 10.6245640 \\
\hline $\mathbf{F}$ & -3.7834880 & -12.2210747 & 10.0959803 \\
\hline $\mathbf{F}$ & -1.6202517 & -10.9143025 & 10.2569919 \\
\hline $\mathbf{F}$ & 0.5154184 & -9.6594497 & 10.4538071 \\
\hline $\mathbf{F}$ & 2.6544943 & -8.4110253 & 10.6682394 \\
\hline $\mathbf{F}$ & 4.8508886 & -7.1755950 & 10.9486168 \\
\hline $\mathbf{F}$ & 6.1569145 & -5.0454091 & 11.9063570 \\
\hline $\mathbf{C}$ & 4.8388428 & -5.1460016 & 12.0495467 \\
\hline $\mathbf{C}$ & 4.1597628 & -6.2201990 & 11.5705888 \\
\hline $\mathbf{C}$ & 2.7418641 & -6.3351390 & 11.7375220 \\
\hline $\mathrm{C}$ & 2.0101592 & -7.4255923 & 11.2960639 \\
\hline
\end{tabular}




\begin{tabular}{|llll|}
$\mathbf{C}$ & 0.6158263 & -7.5540800 & 11.4888707 \\
\hline $\mathbf{C}$ & -0.1243297 & -8.6677498 & 11.0735336 \\
\hline $\mathbf{C}$ & -1.5022553 & -8.7965939 & 11.2890458 \\
\hline $\mathbf{C}$ & -2.2541555 & -9.9224957 & 10.8858544 \\
\hline $\mathbf{C}$ & -3.6137220 & -10.0525402 & 11.1182964 \\
\hline F & 4.8702585 & -3.0715172 & 13.1756125 \\
\hline $\mathbf{C}$ & 4.1555566 & -4.0963847 & 12.7209751 \\
\hline C & 2.8095142 & -4.1459861 & 12.8929495 \\
\hline F & 2.2186184 & -3.1355094 & 13.5304975 \\
\hline $\mathbf{C}$ & 2.0422836 & -5.2575332 & 12.4168853 \\
\hline F & 0.6718539 & -5.3600277 & 12.5888089 \\
\hline F & 0.0329505 & -4.3632203 & 13.2037083 \\
\hline $\mathbf{C}$ & -2.1016074 & -5.6072945 & 12.9854445 \\
\hline $\mathbf{C}$ & -0.0824243 & -6.4748094 & 12.1596421 \\
\hline $\mathbf{C}$ & -1.4617879 & -6.5979326 & 12.3643925 \\
\hline $\mathbf{C}$ & -2.1987530 & -7.7186192 & 11.9635362 \\
\hline $\mathbf{C}$ & -4.3102043 & -8.9741183 & 11.7990255 \\
\hline F & -3.5869322 & -7.8578312 & 12.1846898 \\
\hline C & -4.2307927 & -6.8673865 & 12.8051882 \\
\hline C & -5.7142439 & -9.1193625 & 12.0386470 \\
\hline F & -6.3817310 & -10.2351598 & 11.6474168 \\
\hline F & -7.6845564 & -10.3723649 & 11.8737450 \\
\hline & -6.4014332 & -8.1606898 & 12.6601568 \\
\hline
\end{tabular}


TD-DFT results for the vertical excitation energies of the previously given dimers are reported in Tables S6 to S8. The results reinforce the importance of long-range corrections to achieve a reasonable description of the charge transfer state on the level of TD-DFT. The results are in good qualitative agreement with the results of our NOFG ansatz given in tables 1 to 3 of the main text.

Table S6: Excitation energies $E_{\mathrm{ex}}$ of the dimer in the end-on molecular orientation. The three given excitation energies correspond to the lowest excitations being localized either on PFP or PEN, or to the CT excitation. The structure was optimized on the level of DFT using the B3LYP functional, the excitation energies were calculated on the level of TD-DFT using either the B3LYP, or the CAM-B3LYP functional showing the importance of long-range correction. All energies are given in $\mathrm{eV}$.

\begin{tabular}{|c|c|c|}
\hline 0 & B3LYP & CAM-B3LYP \\
\hline$E_{\text {ex,PFP }}$ & 1.68 & 2.30 \\
\hline$E_{\text {ex,PEN }}$ & 1.88 & 2.50 \\
\hline$E_{\text {ex,CT }}$ & 0.82 & 2.35 \\
\hline
\end{tabular}

Table S7: Excitation energies $E_{\mathrm{ex}}$ of the dimer in the herringbone molecular orientation. The three given excitation energies correspond to the lowest excitations being localized either on PFP or PEN, or to the CT excitation. The structure was optimized on the level of DFT using the B3LYP functional, the excitation energies were calculated on the level of TD-DFT using either the B3LYP, or the CAM-B3LYP functional showing the importance of long-range correction. All energies are given in $\mathrm{eV}$.

\begin{tabular}{|c|c|c|}
\hline$\square$ & B3LYP & CAM-B3LYP \\
\hline$E_{\text {ex,PFP }}$ & 1.67 & 2.29 \\
\hline$E_{\text {ex,PEN }}$ & 1.87 & 2.50 \\
\hline$E_{\text {ex,CT }}$ & 0.92 & 1.88 \\
\hline
\end{tabular}

Table S8: Excitation energies $E_{\text {ex }}$ of the dimer in the $\pi$-stacked molecular orientation. The three given excitation energies correspond to the lowest excitations being localized either on PFP or PEN, or to the CT excitation. The structure was optimized on the level of DFT using the B3LYP functional, the excitation energies were calculated on the level of TD-DFT using either the B3LYP, or the CAM-B3LYP functional showing the importance of long-range correction. All energies are given in $\mathrm{eV}$.

\begin{tabular}{|c|c|c|}
\hline$\square$ & B3LYP & CAM-B3LYP \\
\hline$E_{\text {ex,PFP }}$ & 1.67 & 2.28 \\
\hline$E_{\text {ex,PEN }}$ & 1.87 & 2.51 \\
\hline$E_{\text {ex,CT }}$ & 1.06 & 1.92 \\
\hline
\end{tabular}

